\title{
DAS VERHALTEN VON BLATTFEDERSEISMOGRAPHEN BEI FREIER UND ERZWUNGENER SCHWINGUNG
}

\author{
voN O. FörTSCH
}

Einleitung. - In der reophysikalischen Literatur findet man eine grosse Anzahl von Arlueiten, die sich theoretisch und experimentell mit dem. Verhahten von Frschütterungsmessern jeder Art und mit der Diskussion der von diese erhahtenen Aufzeichnungen befassen. Bei theoretischen Betrachtungen wird der Ansatz der Kräfte so gestaltet, dass er auf die Schwingungsdifferentialgleichung, $d$. h. dass er auf eine lineare Differentialgleichung zweiter Ordnnng mit konstanten Koeffizienten führt. M. Rössig e r (1, 2) zeigle jedoch durch Rechnung und Experiment, dass ein Blattfederseismograph oder, um es noch allgemeiner auszudrücken, eine von einer Blattfeder einseitir gehaltene Masse nicht den Voraussetzungen grenügt, die zu einer Differentialgleichung ol,igen Types führen. Er heschränkte sich daranf, nachzuweisen, dass eine derartig aufgehängte Masse nicht, wie es sich aus der Schwingungsdifferentialgleichung ergilt, eine, sondern zwei Eignenschwingungen besitzt. Die Richtigkeit seiner Uherlegrungen konnte er an Modellversuchen nachweisen.

Bei der Anwendung der Formeln von $R \ddot{c} s \mathrm{sig}$ e $r$ auf die trans. portablen Blattfederseismographeu, wie sie nach den Angalsen von Wi echert und $A \mathrm{n}$ gre $\mathrm{n}$ he is ter $(\because, 4)$ geloaut worden sind. ergalsen sich fïr die zweite Eigenschwingung Werte, die weit ausserhall, des Berciches lagen, der für die Seismik von Interesse ist. Danach konnte man auch weiterhin diese Erschiitterungsmesser als einfache Schwingungssysteme betrachten. Genauere Untersuchungen zeigten jedoch, dass das Verhalten dieser Instrumente doch merklich ron dem ahweicht, was nach der gewönnlichen Schwingungedilferentialgleichung zu erwarten ist. Dies ausserte sich hesonders hei den Eichungen auf Schüttehtischen iu Bereich der höheren Frequenzen. Diese Diskrepanz veranlasste den Verfasser, das Verhalten dieser Seismographen nïlier zu studieren. In dieser Abbandlung soll über die Untersuchungsergelnnisse berichtet werden. Im ersten Teil wird die Theorie der Blattfederscismographen dargestellt werden. Sie stülzt sich auf 
den Kräfteansatz, den Rössiger in seinen Arbeiten als Ausgangspunkt aufgestellt hat. Er wird hier jedoch durch Hinzufügung eines Dämpfungsgliedes und hei Vertikalseismographen durch Beriicksichtigung des von der Spiralfeder herrihrenden Anteiles der Direktionskraft erweitert. In zweiten Teil wird in Rahnen der Erörterung der experimentellen Ergebnisse vor allem der Einfluss der Klemmung der Blattfedern auf das Verhalten der Seismographen dargelegt. Der Einspannwert und seine Abhängigkeit vom Material der Klemmung wird untersucht.

\section{Teil. Die Theorie der Blattfederseismograplilin.}

1. Aufstellung der Differentialgleichungen. - Sie griindet sich, wie schon erwahnt, auf den Gedankengang von $R$ ös ig e r. Um gleich den allgemeinen Fall zu behandeln, wird die Theorie des Vertikalseismographen, dessen Masse von einer Spiralfeder grehalten wird, dargestellt. Die Theorie des Horizontalseismographen kann dann als ein Spezialfall aufgefasst werden. Fig. 1 zeigt das Schema des Vertikalseismographen. In die Figur ist ein Achsenkreuz so hineingelegt, dass dessen Ursprung in die Klemmstelle der Blattfeder am Stativ
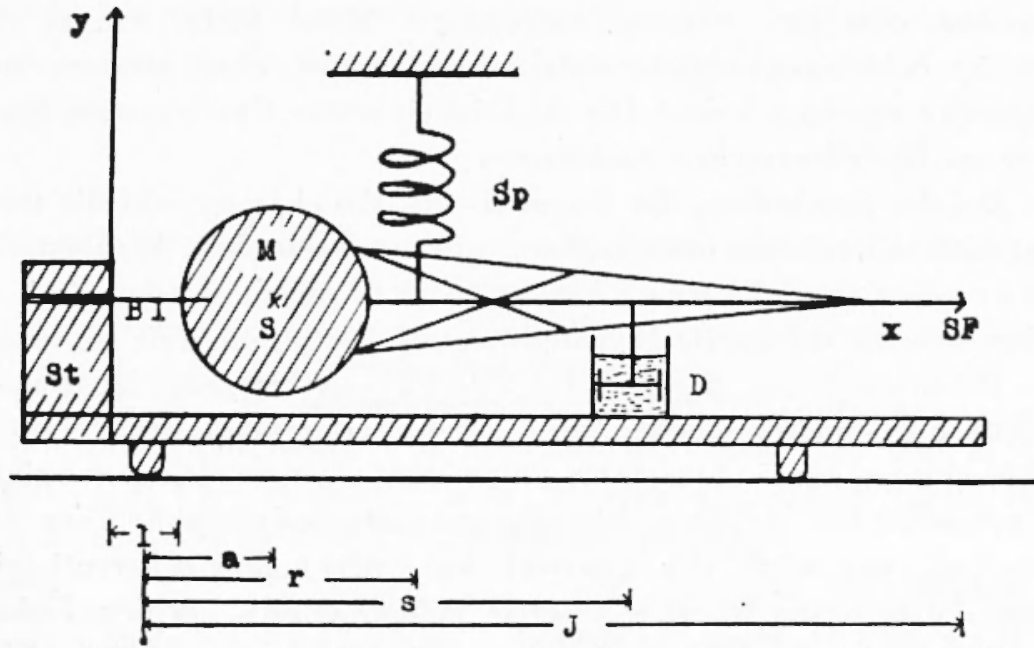

Fig. 1 - Schema eines Vertikalseismographen. $S t=$ Stativ nit Klemmvorrichtung für die Blattfeder; $M=$ Masse des Seismographen; $S=$ deren Schwerpunkt; $S_{p}=$ Spiralfeder; $D=$ Dämpfung; $S F=$ Schreihfeder hezw. Ende des an der Masse befestigten starren Hebels; $B l=$ Blattfeder; $l=$ freie Länge der Blattfeder; $a=$ Abstand Blattfedermitte-Schwerpunkt; $r=$ Abstand Blattedermitte-Angriffspunkt der Spiralfeder; $s=$ Abstand Blattfedermitte-Angriffspunkt der Dampiung; $J=$ Abstand Blattfedermitte-Schreibfeder. 
fältt. Die $x$-Achse verläuft horizontal und fällt mit der Nullage des Systems zusammen. Die y-Achse steht senkrecht darauf und weist nach oben.

Bei einer Auslenkung des Apparates ans seiner Gleichgewichtslage wird, solange es sich um kleine Beträge handelt, die y-Koordinate eines Punktes $x>l$ durch die Beziehung gegehen:

$$
y=y_{1}+(x-l) \varphi,
$$

wolei $y_{1}$ die Auslenkung des Blattfederendes $x_{1}=l$ und y der Winkel ist, den die Tangente an das Blattfederende mit der $\boldsymbol{x}$-Acbse bildet.

Vernachlässig̨ man die Masse der Blattfeder gegenülser der des Seismographen, was hier olıne Bedenken immer erlault ist, so ist hei einer Biegung der Blattfeder nach den Lehren der Elastizitätstheorie das Moment der Normalspannung an irgendeiner Stelle $x<l$ gleich der Summe der Momente der äusseren Kräfte. Es gilt folgende Beziehung:

$$
+N=-B \frac{d^{2} y}{d x^{2}}=+\sum M
$$

$B$ hedeutet hierbei die Steifigkeit der Blattferler. Sie ist das Produkt aus dem Elastizitïtsmodul $E$ und dem äquatorialen Trägheitsmoment I. $B=E \cdot I$. Für eine Feder rechteckigen Querschnittes ist $I==d^{3} h / 12$ ( $d=$ Dicke, $h=$ Bıcite der Feder). Das Minuszcichen muss in Gl. [1] geselzt werden, da einer positiven Krümmung ein negatives Moment entspriclit. Dic Bedeutung der in den folgenden Ausführungen benutzten Buchstaben $l, a, r, s$ und $J$ ist aus der Fig. $l$ zu ersehen. Die Momente der angreifenden Kriafte sind:

a) Das Moment der Trïgheitskraft der Masse des Schwingungssystems gegeniiher Drehung.

$$
M_{1}=K_{\omega} \frac{d^{*} \varphi}{d t^{2}} ;
$$

$K_{t 1}$ stellt das Trägheitsmoment der Seismographenmasse bezogen auf den Schwerpunkt dar. Die Gesamtmasse des Schwingungssystems wollen wir mit $M$ bezeichnen.

b) Das Trägheitsmoment der Masse gegenüher Translation.

$$
M_{\mathrm{r}}=M \frac{d^{2} y_{\mathrm{a}}}{d t^{2}}\left(a+\frac{l}{2}-x\right)=U\left(\frac{d^{2} y_{1}}{d t^{\circ}}+\left(a-\frac{l}{2}\right) \frac{d^{2} \varphi}{d t^{2}}\right)\left(a+\frac{l}{2}-x\right) ;
$$


c) Das Moment der Dämpfungskraft.

$$
M_{\mathrm{d}}=2 \varrho \frac{d y_{\mathrm{t}}}{d t}\left(s+\frac{l}{2}-x\right)=2 \varrho\left(\frac{d y_{1}}{d t}+\left(s-\frac{1}{2}\right) \frac{d_{\mathrm{r}}}{d t}\right)\left(s+\frac{1}{2}-x\right) ;
$$

Hierbei berleutet $2 \emptyset$ die Kraft, die nötiır ist, um den Dämpfungskolben mit der Geschwindigkeit $1 \mathrm{~cm} / \mathrm{s} z u$ bewegen.

d) Das Moment der Ruickstellkraft der Spiralfeder.

$$
M_{c}=c y_{r}\left(r+\frac{l}{2}-x\right)=c\left(y_{1}+\left(r-\frac{l}{2}\right) p\right)\left(r+\frac{l}{2}-x\right)
$$

$c$ ist die Rückstellkraft, die einer Federverlangerung von 1 cm entspricht.

e) Das Moment, das von Krïten hervorgerufen wird, die von aussen an Schwingungssystem angreifen. In den meisten Fïlen sind diese Kräfte Träglıeitskräfte, die bei Erschütterungen des Bodens, und damit auch des Stativs des Seismographen, im Schwerpunkt der Masse $M$ angreifen. (Hier ist rorausqesetzt, dass das Koordinatensystem im Stativ verankert ist und daher die Ersehüterungen mitmachtl. Wird mit $\eta(t)$ der Amplitudenverlauf der Erschütterungr hezeichnet, so erhalt man für das Moment der ausseren Kräife $f(t)$ :

$$
f(t)=W\left(a+\frac{l}{2}-x\right) \frac{d^{*} \eta}{d t^{i}}
$$

Durch Einsetzen der Ausdriicke a. bis e. in G1. [1] erhalıen wir:

$$
\begin{aligned}
& -B \frac{d^{2} y}{d x^{2}}=K_{=} \frac{d^{2} \varphi}{d t^{2}}+. M\left(\frac{d^{2} y_{1}}{d t^{2}}+\left(a-\frac{l}{2}\right) \frac{d^{2} \varphi}{d t^{2}}\right)\left(a+\frac{l}{2}-v\right)+{ }_{2}\left(\frac{d y_{1}}{d t}+\right. \\
& \left.+\left(s-\frac{l}{2}\right) \frac{\left.d_{m}\right)}{d t}\right)\left(s+\frac{l}{2}-x\right)+c\left(y_{1}+\left(r-\frac{l}{2}\right) \varphi\right)\left(r+\frac{l}{2}-x\right)+ \\
& +W\left(a+\frac{l}{2}-x\right) \frac{d^{2} \eta}{d t^{2}} \text {; }
\end{aligned}
$$

Durch Ordnen der Glieder greht Gl. [1a] iber in:

$$
\begin{gathered}
-B \frac{d^{2} y}{d x^{2}}=\left[K_{0}+M\left(a-\frac{l}{2}\right)\left(a+\frac{l}{2}-x\right)\right] \frac{d^{\circ} \psi^{2}}{d t^{2}}+20\left(s-\frac{l}{2}\right)\left(s+\frac{l}{2}-\right. \\
-x) \frac{d p}{d t}+c\left(r-\frac{l}{2}\right)\left(r+\frac{l}{2}-x\right)\left(p+M\left(a+\frac{l}{2}-x\right) \frac{d^{2} y_{1}}{d t^{2}}+2 \varrho(s+\right. \\
\left.+\frac{l}{2}-x\right) \frac{\frac{d y_{1}}{d t}+c\left(r+\frac{l}{2}-x\right) y_{1}+M\left(a+\frac{l}{2}-x\right) \frac{d^{2}}{d t^{2}} ;}{[1 b]}
\end{gathered}
$$


Um die Werte für $y_{1}$ und q, die gesucbt sind, ermitteln zu können, muss Gl. [1h] ein- und zweimal nach $x$ integriert werden.

$$
\begin{aligned}
& -B \frac{d y}{d x}=\left[K_{0}+M\left(a-\frac{1}{2}\right)\left(a+\frac{1}{2}-\frac{x}{2}\right)\right] x \frac{d^{2}}{d t^{2}}+2\left(s-\frac{l}{2}\right) \\
& \left.\left(s+\frac{1}{2}-\frac{x}{2}\right) x \frac{d(p)}{d t}+c i r-\frac{1}{2}\right)\left(r+\frac{1}{2}-\frac{x}{2}\right) x\left(p+M\left(a+\frac{l}{2}-\right.\right. \\
& \left.-\frac{x}{2}\right) x \frac{d^{2} y_{1}}{d t^{2}}+2_{0}\left(s+\frac{1}{2}-\frac{x}{2}\right) x \frac{d y .}{d t}+c\left(r+\frac{1}{2}-\frac{x}{2}\right) x y_{1} \\
& +M\left(a+\frac{1}{2}-\frac{x}{2}-x \frac{d^{2} \|}{d t^{2}}+C_{1}\right. \text {; } \\
& -B y=\left[K_{0}+M\left(a-\frac{l}{2}\right)\left(a+\frac{l}{2}-\frac{x}{3}\right)\right] \frac{x^{2}}{2} \frac{d^{2} \uparrow}{d t^{2}}+2 \rho\left(s-\frac{l}{2}\right) \\
& \left(s+\frac{l}{2}-\frac{x}{3}\right) \frac{x^{2}}{2} \frac{d \varphi}{d t}+c\left(r-\frac{l}{2}\right)\left(r+\frac{l}{2}-\frac{x}{3}\right) \frac{x^{2}}{2} \varphi+1\left(a+\frac{l}{2}-\right. \\
& \left.-\frac{x}{3}\right) \frac{x^{2}}{2} \frac{d^{0} y_{i}}{d t^{2}}+Z_{\varrho}\left(s+\frac{l}{2}-\frac{x}{3}\right) \frac{x^{2}}{2} \frac{d y_{i}}{d t}+c\left(r+\frac{l}{2}-\frac{x}{3} i \frac{x^{z}}{2} y_{1}\right. \\
& +\boldsymbol{M}\left(\boldsymbol{a}+\frac{l}{2}-\frac{x}{3}\right) \frac{x^{\circ}}{2} \cdot \frac{d^{\prime \prime \eta}}{d l^{2}}+C_{1} x+C_{2} ;
\end{aligned}
$$

Die Konstanten $C_{1}$ und $C_{22}$ lassen sich aus den Randbedingungen bestimmen. Für den am Stativ cing̣eklemmten Blattfederanfang muss gelten:

$$
\text { fïr } x=O: \frac{d y}{d x}=O \text { und } y=O ;
$$

Darau: erg̣ibt sich:

$$
C_{1}=O \text { und } C_{s}=O \text {; }
$$

Un die Werte $y=\frac{d y}{d x}{ }_{\mathrm{x}=1}$ und $y_{1}$ zu erhalten, ist in Gl. [2a] und [2b] fiir $x=l$ einzusetzen. In den folgenden Gleichungen sind leide Sciten gegenüber Gl. [2a] und [2b] durch $l$ bezw. $l^{2 / 2}$ dividiert worden.

$$
\begin{gathered}
-\frac{B}{l} \varphi=\left[K_{0}+M\left(a-\frac{l}{2}\right) a\right] \frac{d^{\prime \prime} \varphi}{d t^{2}}+2 \varrho\left(s-\frac{l}{2}\right) s \frac{d \varphi}{d t}+c\left(r-\frac{l}{2}\right) r \varphi \\
+M a \frac{d^{2} y_{1}}{d t^{2}}+2 \varrho s \frac{d y_{1}}{d t}+c r y_{\mathrm{i}}+M a \frac{d^{2} \eta}{d t^{2}} ;
\end{gathered}
$$




$$
\begin{aligned}
& -\frac{2 B}{i^{2}} y_{1}=\left[K_{0}+M\left(a-\frac{l}{2}\right)\left(a+\frac{l}{6}\right] \frac{d^{2} \varphi}{d t^{2}}+2 \varrho\left(s-\frac{l}{2}\right)\left(s+\frac{l}{6}\right) \frac{d m}{d t}+\right. \\
& +c\left(r-\frac{l}{2}\right)\left(r+\frac{l}{6}\right) \varphi+M\left(a+\frac{l}{6}\right) \frac{d^{2} y_{1}}{d t^{2}}+2 \varrho\left(s+\frac{l}{6}\right) \frac{d y_{1}}{d t}+ \\
& +c\left(r+\frac{l}{6}\right) v_{i}+M\left(a+\frac{l}{6}\right) \frac{d^{2} \eta}{d t^{2}} ;
\end{aligned}
$$

Durch Zusammenziehen der Glieder erhalten wir die endgiiltigen Differentialgleichungen:

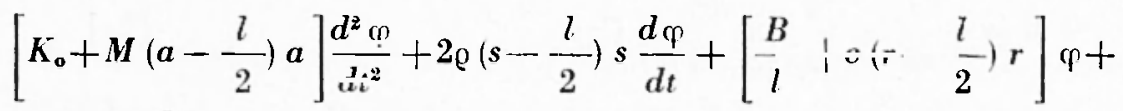

$$
\begin{aligned}
& +M a \frac{d^{2} y_{1}}{d t^{2}}+20 s \frac{d y_{1}}{d t}+c r y_{1}=-M a \frac{d^{2} \eta}{d t^{2}} \quad ; \\
& {\left[K_{\mathrm{o}}+M\left(a-\frac{l}{2}\right)\left(a+\frac{l}{6}\right)\right] \frac{d^{2} \varphi}{d t^{2}}+2 \varrho\left(s-\frac{l}{2}\right)\left(s+\frac{l}{6}\right) \frac{d \varphi}{d t}+c\left(r-\frac{l}{2}\right)} \\
& \left(r+\frac{l}{6}\right) \varphi+M\left(a+\frac{l}{6}\right) \frac{d^{2} y_{1}}{d t^{*}}+2 \varrho\left(s+\frac{l}{6}\right) \frac{d y_{1}}{d t}+\left[\frac{2 B}{l^{2}}+c\left(r+\frac{l}{6}\right)\right] y_{1} \\
& =-M\left(a+\frac{l}{6}\right) \frac{d^{2} \eta}{d t^{2}} ;
\end{aligned}
$$

Der wesentliche Inhalt dieser Gleichungen tritt deutlicher in Erscheinung, wenn man folgende Koordinatentransformation einführt:

$$
y_{1}=y^{\prime}+\frac{l}{2} \varphi
$$

Die Bewegungen des Schwingungssystems werden damit durch die Bewegungen $y^{\prime}$ des zum starren System gehörigen Punktes mit der Abszisse $x=l / 2$ und den Tangentenwinkel $\varphi$ beschrieben.

$$
\begin{aligned}
& \left(K_{\mathrm{o}}+M a^{2}\right) \frac{d^{2} \varphi}{d t^{2}}+2 \varrho s^{2} \frac{d \varphi}{d t}+\left(\frac{B}{l}+c r^{2}\right) \varphi+b a \frac{d^{2} y^{\prime}}{d t^{2}}+2 \varrho s \frac{d y^{\prime}}{d t}+ \\
& +\operatorname{cr} y=-M a \frac{d^{2} n}{d t^{2}}
\end{aligned}
$$

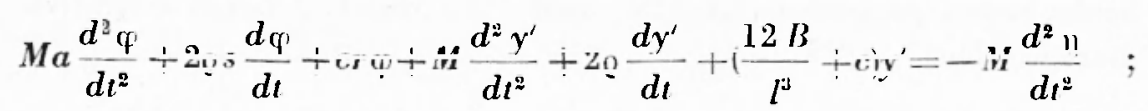

Die Gl. [5a] und [5b] stellen die Differentialgleichungen zweier gekoppelter Schwingungssysteme in der allgemeinsten Form dar. Das eine ist ein Drehschwingungssystem. Die Drehungen erfolgen um den 
Punkt mit den Koordinaten $x=l / 2$ und $y=O$. Das andere System vollführt Translationsschwingungen. Ein Blattfederseismograph kann als ein schwingungsfähiges Gehilde mit zwei Freiheitsgraden und daher anch mit zwei Eigenschwingungen angesehen werden. Diese beiden sind jedoch durch die Masse, die Dämpfung und die Spiralfeder sehr stark miteinander gekoppelt. Daher treten die Eigenschwingungen nic in Erscheinung. Es kommt ihnen keine praktische Bedeutung zu. Bedeutungsvoll sind nur die Koppelfrequenzen.

In den folgenden Ahschnitten soll die Lösung der Differentialgleichungen [5] fïr den homogenen Fall $(\|=O)$ und fiir den inho. mogenen Fall einer periodischen Anregung behandelt werden. Die Lösungen lassen sich hier wegen der grossen Ähnlichkeit der sich entsprechenden Kocflizienten olıne grosse Mühe finden.

2. Losung der homogenen Differentialgleichungen. - Die Ausgangsgleichungen erhalten wir aus Gl. [5a] und [5h] indem wir $\eta=O$ sctzen. Gl. [6a] entsteht durch Suhtraktion der mit a multiplizierten Gl. [5l)] von $\mathrm{Gl}$. [5a].

$$
\begin{aligned}
& K_{\mathrm{o}} \frac{d^{2} \varphi}{d t^{*}}+2 \Omega s(s-a) \frac{d \varphi}{d \imath}+\left(\frac{B}{l}+c r(r-a)\right)\left(\rho+2 \varrho(s-a) \frac{d y^{\prime}}{d \ell}+\right. \\
&+\left(c(r-a)-\frac{12 B a}{l^{3}}\right) y^{\prime}=0 ; \quad[6 \mathrm{a}] \\
& M a \frac{d^{2} \varphi}{d t^{2}}+2 \varrho s \frac{d \varphi}{d t}+\operatorname{cr}\left(\varphi+M \frac{d^{2} y^{\prime}}{d t^{2}}+2 \varrho \frac{d y^{\prime}}{d t}+\left(\frac{12 B}{l^{3}}+c\right) y^{\prime}=0 ;\right.
\end{aligned}
$$

Dic Losungen setzen wir wie iiblich in der Form $q=$ iphis und $v_{1}=Y e^{h}$ an. Nach Bildung der ersten und zweiten Ahleitungen dieser Ausdrücke nach der Zeit t, Einsetzen derselben in G1. [6a] und [61)] und nach Division mit e $M$ crgeben sich:

$$
\begin{gathered}
\text { Ф }\left[K_{0} \hat{\imath}^{3}+2 \varrho s(s-a) \lambda+\left(\frac{B}{l}+c r(r-a)\right)\right]+Y[2 \varrho(s-a) \lambda+ \\
\left.+\left(c(r-a)-\frac{12 B a}{\iota^{3}}\right)\right]=0 \\
\text { Ф }\left[M a \lambda^{2}+2_{\varrho} s \lambda+c r\right]+Y\left[M \lambda^{2}+2 \varrho \lambda+\left(\frac{12 B}{l^{3}}+c\right)\right]=0
\end{gathered}
$$

Sieht man von den trivialen Lösungen $\Phi=O$ und $\mathrm{Y}=O$ ab, so 
können die GI. [7a] und [7h] nur befriedigt werden, wenn der Wert der Koeffizientendeterminante versehwindet. Aus dieser Bedingung lïsst sich $\lambda$ bercchnen. Gleichzeitig folgt aluer noch, dass W und $Y$ keine von einander unah,hängige Grossen sein können. Darauf greifen wir später wieder zuriick.

Die Ausrechnung der Determinante führt auf cine Gleichung vierten Grades in i..

$$
\begin{gathered}
M K_{\mathrm{o}} \lambda^{4}+2 \varrho\left(K_{\mathrm{o}}+M(s-a)^{2}\right) \nu^{3}+\frac{12 B}{l^{3}}\left[K_{\mathrm{o}}\left(1+\frac{c^{*}}{12 B}\right)+M\left(a^{2}+\frac{F}{12}+\right.\right. \\
\left.\left.+\frac{c l^{3}}{12 B}(r-a)^{2}\right)\right] \lambda^{2}+2 \varrho \frac{12 B}{l^{3}}\left[s^{2}+\frac{l^{2}}{12}-\frac{c l^{3}}{12 B}(r-s)^{2}\right] \lambda+\frac{12 B}{l^{3}}\left(\frac{B}{l}+\right. \\
\left.+c\left(r^{2}+\frac{l^{2}}{12}\right)\right)=0
\end{gathered}
$$

orler

$$
\begin{gathered}
\lambda^{2}+20 \frac{K_{\mathrm{o}}+\boldsymbol{M}(s-a)^{2}}{M K_{\mathrm{o}}} \lambda^{3}+\frac{12 B}{l^{3} M K_{\mathrm{o}}}\left[K_{\mathrm{o}}\left(1+\frac{c l^{3}}{12 B}\right)+M\left(a^{2}+\frac{l^{2}}{12}+\right.\right. \\
\left.\left.+\frac{c l^{3}}{12 B}(r-a)^{2}\right)\right] i^{2}+2 \varrho \frac{12 B}{l^{3} M K_{0}}\left(s^{2}+\frac{l^{2}}{12}+\frac{c_{t^{3}}}{12 B}(r-s)^{2}\right) i+\frac{12 B}{l^{3}} \\
\left(\frac{B}{l}+c\left(r^{2}+\frac{l^{2}}{12}\right)\right)=0
\end{gathered}
$$

In Gl. [8"] führen wir grleich einige Vereinfachungen durch, indem wir praktisch bedeutungslose Glieder weglassen. Bei allen Scismographen ist $l / \sqrt{12}$ klein gegen $a, s$ und $r$. Das Verhälnis ist in allgemeinen kleiner als 1:50. Ungünstigenfalls wird es 1:10. Daher kann bedenkenlos $l / 12$ als Summand in den Koeffizienten von Gl. $\left[8^{\prime \prime}\right]$ gestrichen werden. Gewöhnlich ist auch $\mathrm{cl}^{3 / 12} B$ klein geurcon 1 . Bei allen Horizontalpendeln, die olme Spiralfeder arlseiten, ist $c$ von vorneherein Null. Bei den Vertikalapparaten mit Spiralfeder ist man aus technischen Gründen gezwungen die Grösen $B$. $l$ und $c$ so zu wäh. len, dass $c l^{\prime \prime} 12 B$ klein gegen 1 wird. Einerseits muss nämlich $B$ so gross und $l$ so klein gremacht werden, dass dic Masse des Seismorraploen eine sichere Fül)rung loat. Andererseits darf $c$ ein loestimmtes Mass nicht überschreiten, da die Spiralfeder nur zuverlassig arbeiten kann, wenn sie durch die Masse merklich gereckt wird. In allgemeinen liegt der Wert ron $c l^{*} 12 \mathrm{~B}$ zwischen $10^{-3}$ und $10^{5}$. Um daher die Chersichtlichkeit der Formeln durch praktisch hedeutungslose 
Ausdrücke nicht zu stören, werden in Gl. [8"] auch die Summanden $\mathrm{cl}^{\circ} / 12 \mathrm{~B}$ vernachlässigt. Damit geht die Gleichung ïher in:

$$
\begin{aligned}
& \lambda^{4}+2 \varrho \frac{K_{0}+M(s-a)^{2}}{M K_{0}} ; 3 \perp-\frac{12 B}{l^{3} M K_{0}} ; K_{0}: M a^{2} ?^{2}: 2 \frac{12 B}{l^{3} M K_{0}} s^{2} \lambda+ \\
& +\frac{12 B}{l^{3} M K_{1}}\left(\frac{B}{l}+c r^{2}\right)=0
\end{aligned}
$$

Eine exakte Bestimmung der Wurzehn von Gl. [8] läst sich schwerlich herloeiführen. Zu praktisch ausreichenden Näherungslösungen kommt man aher, wenn man herücksichtigt, dass der Ausdruck $12 B\left(K_{0}+M a^{2}\right) l^{3} M K_{0}$ sehr gross gegen 1 ist. Sein Wert liegt bei den grehräuchlichen Seismographen wohl immer über 10\%. Dies hedeutet, dass die Alssolutheträge von zwei Wurzeln der Gl. [8] gross sein missen gregenüher denen der heiden anderen Wurzeln.

Da in Gl. [8] sämtliche Koeffizienten positiv sind, sind die Lösungen alle komplex. Wir setzen sie in der für Schwingungsprobleme allrremein ïblichen Form an:

$$
\begin{aligned}
& \lambda_{1}=-\delta_{1}+i \sqrt{(1)_{1}^{2}-\delta_{1}^{2}} \quad ; \quad \lambda_{0}=-\delta_{1}-i \sqrt{(1)_{1}{ }^{2}-\delta_{1}^{2}} ; \\
& \lambda_{3}=-\delta_{2}+i \sqrt{\omega_{0}^{2}-\delta_{2}^{2}} \quad ; \quad \lambda_{4}=-\delta_{2}-l \sqrt{\omega_{0}^{2}-\delta_{2}^{2}} ;
\end{aligned}
$$

Da $\left(\lambda-\lambda_{1}\right)\left(\lambda-\lambda_{9}\right)\left(\lambda-\lambda_{3}\right)\left(i-i_{4}\right)=O$ sein muss, ergiht sich durch Ausmultiplizicren:

$$
\begin{aligned}
\lambda^{4}+2\left(\delta_{1}+\delta_{2}\right) \lambda^{3}+ & \left(\omega_{1}{ }^{2}+\left(\omega_{2}{ }^{2}+4 \delta_{1} \delta_{2}\right) \lambda^{2}+2\left(\delta_{1} \omega_{2}{ }^{2}+\delta_{2}(1)_{1}^{2}\right) \lambda_{2}+(1)_{1}{ }^{2}(1)_{2}{ }^{2}\right. \\
& =\lambda^{4}+2 k_{1} \lambda^{3}+k_{2} \lambda^{2}+2 k_{3} \lambda+k_{4}=0
\end{aligned}
$$

Die Koeffizienten $k_{1}, k_{\cdot .}, h_{3}$ und $k_{4}$ werden nur der kürzeren Schreihweise wegen vorihhergehend eingeführt. Sind, wie in Gl. [8], h?, $k$ : und $h_{4}$ gross gegen 1 und $h_{1}$, so muss (1)?" gross gegen $(1)_{1}=, \delta_{1}$ und $\delta_{2}$ sein. Man kann also in $k_{2}, h_{3}$ und $k_{s}$ die Summanden, die $\omega_{2}{ }^{2}$ nicht enthalten, vernachlissigen. Damit kam man die Lösungen sofort hinschreiben

$$
\omega_{0}{ }^{2}=k_{0} ; \delta_{1}=h_{3} / k_{2} ; \omega_{1}{ }^{2}=k_{1} / k_{2} ; \delta_{0}=k_{i}-\delta_{1} ; \quad\left[10^{\prime}\right]
$$


Durch Einsetzen der entsprechenden Werte aus Gl. [8] erhalten wir:

$$
\begin{array}{ll}
\omega_{1}=\sqrt{\frac{B / l+c r^{2}}{K_{0}+M a^{-}}} & \delta_{i}=\frac{\rho s^{2}}{K_{0}+M a^{2}} \\
\omega_{1:}=\sqrt{\frac{12 \bar{B}\left(K_{0}+M a^{2}\right)}{l^{3} M K_{0}}} & \delta_{2}=\frac{\rho\left(K_{0}+M(s-a)^{2}\right)}{M K_{0}}-\frac{\rho s^{2}}{K_{0}+M a^{2}}
\end{array}
$$

Damit sind die Differentialgleichungen [6a] und [6b] näherungsweise gelöst. Die Ausdrücke für q und $y_{\text {I }}$ setzen sich aus den vier Partiallösungen folgendermassen zusammen:

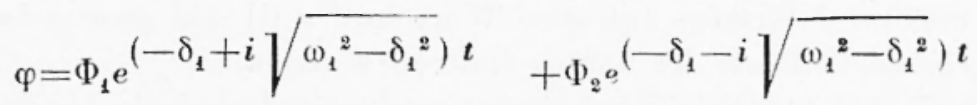

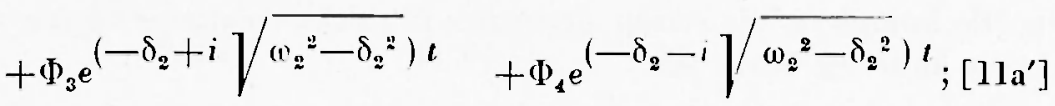

$$
\begin{aligned}
& y_{1}=Y_{1} e^{\left(-\delta_{1}+i \sqrt{\omega_{1}^{2}-\delta_{1}^{2}}\right) t}+Y_{2}\left(-\delta_{1}-i \sqrt{\omega_{1}{ }^{2}-\delta_{1}^{2}}\right) t
\end{aligned}
$$

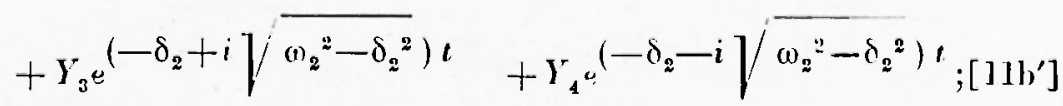

Die Koeffizienten $\Phi_{n}$ und $Y_{11}$ sind konstante Amplitudenwerte. Es wurde oben bereits benerkt, dass sie voneinander abhängig sind. Ihre Verknüpfung wird durch Gl. [7b] festgelegt zu:

$$
Y_{\mathrm{n}}=-\frac{M a \partial_{n}{ }^{2}+2 \rho s \lambda_{n}+c r}{M \prime_{n}{ }^{2}+2 \varrho \rho_{n}+\left(12 b / l^{3}+c\right)} \Phi_{n}=-x_{n} \Phi_{n} \quad ; \quad(n==1,2,3,4)
$$

In Gl. [12] ist in der Klammer in Nenner $c$ als Summand gegenüber $12 B / l^{3}$ vernachlässighar.

Durch Einsetzen der Werte i.. ergeben sich für $\varkappa_{n}$ folgende Aus. drücke :

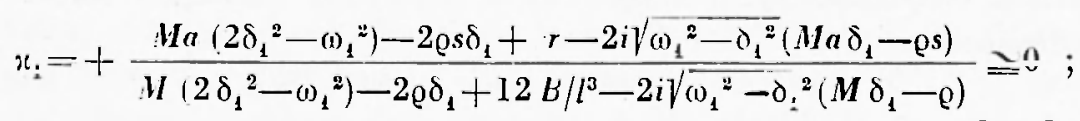




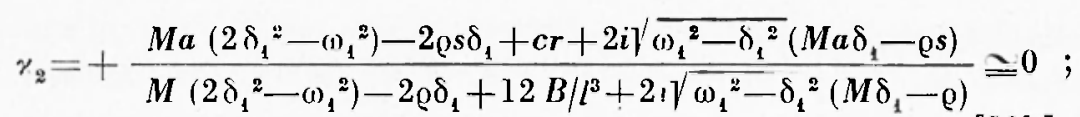

$x_{3}=+\frac{M a\left(2 \delta_{2}{ }^{2}-\omega_{2}{ }^{2}\right)-2 \varrho s \delta_{2}+c r-2 i \gamma^{\prime} \overline{\omega_{2}{ }^{2}-\delta_{2}{ }^{2}}\left(M a \delta_{2}-\emptyset s\right)}{M\left(2 \delta_{2}{ }^{2}-\omega_{2}{ }^{2}\right)-2 \varrho \delta_{9}+12 B / 1^{3}-2 i \sqrt{\omega_{2}{ }^{2}-\lambda_{2}{ }^{2}}\left(M \delta_{2}-\varrho\right)} \cong+a\left(1+\frac{K_{0}}{M a^{2}}\right) ;$

$\gamma_{4}=+\frac{M a\left(2 \delta_{2}^{2}-\omega_{2}^{2}\right)-2 s \delta_{2}+c r+2 i \sqrt{\left(\omega_{2}^{2}-\delta_{2}^{2}\right.}\left(M a \delta_{2}-\varrho s\right)}{M\left(2 \delta_{2}^{2}-\omega_{2}^{2}\right)-20 \delta_{2}+12 B / l^{3}+2 i l^{\prime}\left(\omega_{2}^{2}-\delta_{2}^{2}\left(M \delta_{2}-\varrho\right)\right.} \cong+a\left(1+\frac{K_{0}}{M a^{2}}\right) ;$

Das Auftreten von imaginären Gliedern in den Ausdrüicken für die $x$-Werte besagt, dass in den Partiallösungen die $y$-Schwingung eine Phasenverschiebung gegenüber der ฯ-Schwingung besitzt. Die Koeffizienten der Imaginärteile sind aber klein gegenüber den Absolut. beträgen. Die Phasenverschiebungen sind daher ehenfalls klein. Ihnen kommt keine praktische Bedeutung zu. Sie können weggelassen wer* den. Der Wert der Gl. [13a] und [13b] wird hauptsäcblich durch Glieder, die $B$ als Faktor enthalten, bestimmt. Dasselbe gilt für Gl. [13c] und [13d]. Zu berücksichtigen ist nur, dass $B$ in Ausdruck fïr (1)., auftritt. Durch Einsetzen ergeben sich nach leichten Umfornungen die angegebenen Naherungswerte. Die $z$-Werte haben die Dimension ciner Länge. Auf ihre Bedeutung kommen wir später noch zurïck.

Die Gl. [11 $\left.a^{\prime}\right]$ und [111'] lassen sich folgendermassen umformen:

$$
\begin{aligned}
& \left.\varphi=e^{-\delta_{1} t}\left(f_{1} \cos \sqrt{\omega_{1}{ }^{2}-\delta_{1}{ }^{2} t}-i g_{1} \sin \sqrt{\omega_{1}{ }^{2}-\delta_{1}{ }^{2}}\right)^{\prime}\right) \\
& +e^{-\delta_{2} t}\left(f_{2} \cos \sqrt{\omega_{2}{ }^{2}-\delta_{2}{ }^{2} t}-i g_{2} \sin \sqrt{\omega_{2}{ }^{2}-\delta_{2}{ }^{2} t}\right) ;
\end{aligned}
$$

$\left[11 a^{\prime \prime}\right]$

oder

$$
\varphi=C_{1} e^{-\delta_{1} t} \cos \left(\sqrt{\left.\omega_{1}{ }^{2}-\delta_{1}{ }^{2} t+\gamma_{1}\right)+C_{2}{ }^{-\delta_{2} t} \cos \left(\sqrt{\omega_{2}{ }^{2}-\delta_{2}{ }^{2} t}+\gamma_{2}\right)}\right.
$$

Hierhei sind:

$$
f_{1}=\Phi_{1}+\Phi_{2} ; \quad g_{1}=\Phi_{1}-\Phi_{2} ; \quad f_{2}=\Phi_{3}+\Phi_{4} ; \quad g_{2}=\Phi_{3}-\Phi_{1} ;
$$




$$
\begin{aligned}
& C_{1}=\sqrt{f_{1}^{2}-g_{1}^{2}}=2 \sqrt{\Phi_{1} \Phi_{2}} ; \operatorname{tg} \gamma_{1}=i g_{1} / f_{1} ; \\
& C_{2}=\sqrt{f_{2}^{2}-g_{2}^{2}}=2 \sqrt{\Phi_{3} \Phi_{1}} ; \lg \gamma_{2}=i g_{2} / f_{2} ;
\end{aligned}
$$

Für $y^{\prime}$ gilt das Entsprechende:

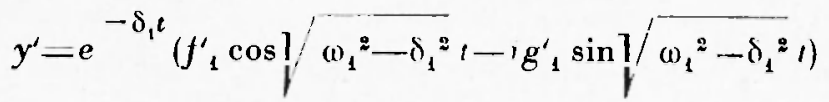

$$
\begin{aligned}
& +e^{-\delta_{2}{ }^{2}}\left(f_{2}^{\prime} \cos \sqrt{\omega_{2}^{2}-\delta_{2}^{2} t}-i g_{2}^{\prime} \sin \sqrt{\left.\omega_{2}^{2}-\delta_{2}^{2} t\right)} ;\right.
\end{aligned}
$$

oder

$$
y^{\prime}=C_{1}^{\prime} e^{-\delta_{1} t} \cos \left(\sqrt{\left.\omega_{1}{ }^{2}-\delta_{1}{ }^{2} t+\gamma_{1}^{\prime}\right)+C_{2}^{\prime} e^{-\delta_{2} t} \cos \left(\sqrt{\omega_{2}{ }^{2}-\delta_{2}{ }^{2} t} t+\gamma_{2}^{\prime}\right)}\right)
$$

Es bedeuten:

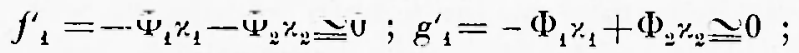

$$
\begin{aligned}
& C_{1}^{\prime}=-\sqrt{f_{1}^{\prime 2}-g_{1}^{\prime}{ }^{2}} \cong 0 ; \operatorname{tg}^{\prime}{ }_{1}^{\prime}=i g_{1}^{\prime} / t_{1}^{\prime} ; \gamma_{1}^{\prime}=\gamma_{1} \text {; } \\
& f_{2}^{\prime}=-\Phi_{3{ }_{3}}-\Phi_{4} \varkappa_{4}=-f_{2} a\left(1+\frac{K_{0}}{M a^{2}}\right) ; g_{2}^{\prime}=-\Phi_{3} \varkappa_{3}+\Phi_{4} x_{4}=-g_{2} a\left(1+\frac{K_{0}}{M a^{2}}\right) ; \\
& C_{2}^{\prime}=-\sqrt{{f_{2}^{\prime}}^{2}-g_{2}^{2}}=-C_{2} a\left(1+\frac{K_{0}}{M a^{2}}\right) ; \operatorname{tg}_{\gamma_{2}^{\prime}}^{\prime}=i g_{2}^{\prime} / f_{2}^{\prime} ; \gamma_{2}^{\prime}=\gamma_{2} ;
\end{aligned}
$$

Damit sind alle Koeffizienten bis auf vier, nämlich $C_{1}, C_{2}, \gamma_{1}$ unel $\gamma_{2}$, die aus den jeweiligen Anfangsbedingungen zu berechnen sind, gegelsen. Die lomogenen Differentialgleichungen sind gelöst. Für dic Bewegung eines Punktes des Schwingungssystems mit der Alszisse $x$ lautet dio Gleichung:

$$
\begin{aligned}
& y_{x}=\left(x-\frac{l}{2}\right) \varphi+y^{\prime}=C_{1}\left(x-\frac{l}{2}\right) e^{-\delta_{1} t} \cos \left(\sqrt{\left(0_{1}^{2}-\delta_{1}^{2} t\right.}+\gamma_{1}\right) \\
& +C_{2}\left(x-\frac{l}{2}-a\left(1+\frac{K_{-2}}{\bar{M} a^{2}}\right)\right) e^{-\delta_{2} t} \cos \left(\sqrt{\omega_{2}^{2}-\delta_{2}^{2}} t+\gamma_{2}\right) ;
\end{aligned}
$$

Bei einem Anstoss der Masse des Seismographen führt dieser zwei Drehschwingungen aus. Die Drehachse der niederfrequenten Schwingung liegt in der Mitte der freien Länge der Blattfeder, $x=l / 2$. Die Eigenfrequenz $v_{1}=\omega_{1} / 2 \pi$ hängt ab l. von der Summe der Drehmo- 
mente, die von den Direktionskräften der Blatt- und der Spiralfeder erzeugt werden, hezogen auf die Drehachse und 2. von dem Trägheitsmoment der Seismographenmasse elenfalls auf die Drehachse $x=l / 2$ bezogen. Die zweite Drehschwingung hat als Drehachse den Punkt $x=a\left(1+K_{0} M a^{2}\right)+l / 2=-\gamma_{3}+l / 2, y=O$. Der Punkt $x=l / 2$ erfährt keine Auslenkung durch die erste und der Punkt $x=a(1+$ $\left.+K_{n} / M a^{2}\right)+l / 2$ keine durch die zweite Schwingung. Dies hat schon Rös siger gefunden. Die richtigen Vorzeichen versehenen Ahstanden der Drehachsen von dem Ahszissenpunkı, auf welchen sich die q- und $y$-Werte heziehen. In unserem Falle ist dies der Punkı $x=l / 2$, da zu ihm die Werte $y^{\prime}$ gehöoren. Deshalh ist auch $\gamma_{1} \cong x_{3}=0 . \gamma_{3}$ und $r_{4}$ sind ungefalor gleich dem Ahstand der beiden Drehachsen. Die Frequenz $v_{2}=(1) .2 / 2-\mathrm{kann}$ man sich hier dargestellt denken als das Produkt aus der Eigenfre-

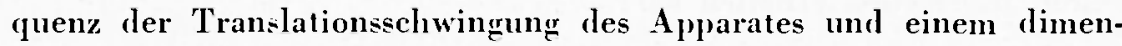
sionslosen Faktor, der von der Gestalt der Masse und der freien Länge der Blattfeder ahliangt.

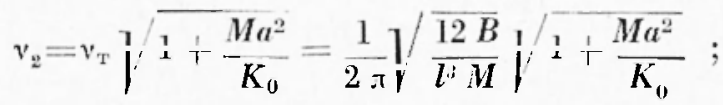

Die Direktionskraft für $*_{r}$ wird praktisch nur durch die Blattfeder hestimmt. Für diese Schwingung ist sie $12 B / l^{3}$, also $12 / l^{2}$ mal grösser als der entsprechende Anteil an $v_{1}$. Die Direktionskraft der Spiralfeder $c$ ist bei den ühlichen Dimensionen der Seismographen gegenüber $12 B /$ " verschwindend klein. Die Spiralfeder hat auf $v_{2}$ keinen Einfluss. Die Drebeigensclowingung und $v_{1}$ sind praktisch identisch, für sie wird der Faktor ungefähr l. Es sei hier nochmals betont, dass $r_{1}$ und $r_{2}$ keine Eigenfrequenzen, sondern Koppelfrequenzen sind.

M. Rösigrer hat sich für den vereinfachten Fall des Scismographen olıne Dämpfung $\theta=O$ und olne Spiralfeder $c=O$ mit der Berechnung der Drehachsen und der Koppelfrequenzen hernüigt. Seine Ergehnisse, zu denen er auf einem anderen Wege der Bcrechnung gelangt ist, sind mit den obigen identiseh. Es braucht daher bezüglich der sich daraus ahzuleitenden Folgernngen nur auf die Veröffentlichungen ron $R \ddot{s s i g e r}$ rerwiesen werden. Auf weitere Einzelheiten werden wir an Ende dieses Teiles der vorliegenden Ahhandlung cingehen.

3. Der Einschuinguorgang. - Der Einschwingrorgang ist hei 
einem gekoppelten Schwingungsgebilde noch viel verwickelter als er schon bei einem einfachen System ist. Un die Rechnungen nicht zu weitschweifend werden zu lassen, wollen wir uns auf den einfachsten Fall beschränken und führen in Gedanken folgenden Versuch aus:

Am Schwerpunkt des Seismographensystems greife eine konstante Kraft $P$ an. Sie lenkt dieses aus seiner Nullage aus. Zur Zeit $t=O$ soll dann die Kraft versehwinden. Wir wollen nun die dadurch verursachte Bewegung des Seismographen untersuchen.

Zur Beschreibung der Bewegung des Systems bedienen wir uns wieder der Grössen ' $q$ und $y^{\prime}$. Solange die Kraft $P$ wirkt, sind beide konstant. Sie lassen sich aus dem Krïfteansatz berechnen.

$$
+B \frac{d^{2} y}{a x^{2}}=P\left(a+\frac{l}{2}-x\right)-c\left(y^{\prime}+r \varphi\right)\left(r+\frac{l}{2}-x\right)
$$

Durch Integration erhalten wir:

$$
\begin{aligned}
& B \frac{d y}{d x}=P\left(a+\frac{l}{2}-\frac{x}{2}\right) x-c\left(y^{\prime}+r y\right)\left(r+\frac{l}{2}-\frac{x}{2}\right) x \\
& B y=P\left(a+\frac{l}{2}-\frac{x}{3} ; \frac{x^{2}}{2}-c\left(y^{\prime}+r(p)\left(r+\frac{!}{2}-\frac{x}{3}\right) \frac{x^{2}}{2}\right.\right.
\end{aligned}
$$

Es interessieren wieder die Werte $\varphi$ und $y$ für $x=1$. Für $d y / d x$ wird $\varphi$ und für $y$ wird $y_{1}=y^{\prime}+\varphi l / 2$ in obigen Gleichungen cingesetzt, während gleichzeitig $x$ durch $l$ ersetzt wird. Subtrahiert man dann noch die obere Gleichung von der unteren und dividiert durch $l ; 6$, so bekommt man folgende Bestimmungsgleichungen für $p$ und $y^{\prime}$ :

$$
\begin{aligned}
& \left(\frac{B}{l}+c r^{2}\right) p+c r y^{\prime}=P a \\
& \operatorname{cr} \varphi+\left(\frac{12 B}{l^{3}}+c\right) y^{\prime}=P
\end{aligned}
$$

Daraus berechnen sich $\uparrow$ und $y^{\prime}$, wenn man die oben diskutierten Vernachlässigungen macht, zu:

$$
\varphi=\frac{P a}{B / l+c r^{2}} ; y^{\prime}=\frac{P l^{3}}{12 B}\left(1-\frac{c r a}{B / l+c r^{2}}\right) \supseteq 0 ;
$$

Diese beiden Beziehungen müssen bis $t=O$ einschliesslich grelten. Das Gleiche muss für die folgenden beiden der Fall sein.

$$
\frac{d p}{d t}=0 ; \frac{d y^{\prime}}{d t}=0 ;
$$


Diese vier Anfangshedingungen dienen zur Berechnung der Konstanten der GI. [15]. Aus GI. [11a"], [11b"], [14a] und [14b] erhalten wir für $t=O$ :

$\varphi=f_{1}+f_{2} ;$

$y^{\prime}=-a\left(1+K_{0} / M a^{2}\right) f_{2}$;

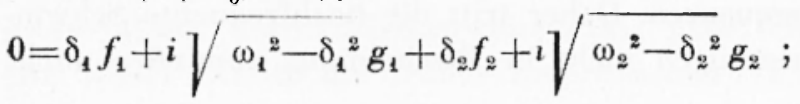

$0=-a \delta_{2}\left(1+K_{0} / M a^{2}\right) f_{2}-\sqrt{\omega_{2}{ }^{2}-\delta_{2}^{2}} a\left(1+K_{0} / M a^{2}\right) i g_{2} ;$

$\delta_{2} f_{2}+\sqrt{\omega_{2}^{2}-\delta_{2}^{2}} i g_{2}=0$;

damit ist auch : $\delta_{1} f_{1}+\sqrt{\omega_{1}^{2}-\delta_{1}^{2}} i g_{1}=0$;

Die Koeffizienten berechnen sich daraus, wenn man noch folgende Abkürzungen benutzt,

$L=a\left(1+K_{0} / M a^{2}\right) ; \quad \varphi_{u}=-y^{\prime} / L ;$

zu:

$$
\begin{gathered}
f_{1}=\varphi-\varphi_{2} ; f_{2}=+\varphi_{2} ; i g_{1}=-\left(\varphi-\varphi_{2}\right) \delta_{1} / \sqrt{\omega_{1}^{2}-\delta_{1}^{2}} ; \\
i g_{2}=-\varphi_{2} \delta_{2} / \sqrt{\omega_{2}^{2}-\delta_{2}^{2}} ; \\
C_{1}=\frac{\alpha_{1}}{\sqrt{\omega_{1}^{2}-\delta_{1}^{2}}}\left(\varphi-\varphi_{2}\right) ; C_{2}=\frac{\omega_{2}}{\sqrt{\omega_{2}^{2}-\delta_{2}^{2}}} \varphi_{2} ; \\
\operatorname{tg}\left(-\gamma_{1}\right)=\frac{\delta_{1}}{\sqrt{\omega_{1}^{2}-\delta_{1}^{2}}} ; \operatorname{tg} \gamma_{2}=\frac{-\delta_{9}}{\sqrt{\omega_{2}^{2}-\delta_{2}^{2}}}
\end{gathered}
$$

$\varphi$. ist der Winkel, unter dem man von der Drehachse der zweiten Schwingung aus die Ordinate $y^{\prime}$ sieht. Die Schreibfeder, hezw. der Endpunkt des mit der Masse starr verbundenen IIcbels der Registriereinrichtung fübrt folgende Bewegung aus:

$$
\begin{aligned}
& y_{j}=\left(\varphi-\varphi_{0}\right) \frac{\omega_{1}}{V^{\prime}\left(_{1}^{2}-\delta_{1}^{2}\right.} J e^{-\delta_{1} t} \cos \left(\sqrt{\omega_{1}^{2}-\delta_{1}^{2} t}-\operatorname{arctg} \frac{\delta_{1}}{\sqrt{\omega_{1}^{2}-\delta_{1}^{2}}}\right) \\
& +\varphi_{s}(J-L) e^{-\delta_{2} t} \cos \left(\sqrt{\omega_{2}^{2}-\delta_{2}^{2} t}-\operatorname{arctg} \frac{\delta_{0}}{\sqrt{\omega_{0}^{2}-\delta_{0}^{2}}}\right) ;
\end{aligned}
$$


In dieser Gleichung ist $\omega_{g} / \sqrt{\omega_{9}{ }^{2}-\delta_{g}{ }^{2}}=1$ gesetzt worden, da der Wert des Quotienten doeh nur wenig davon abweicht. $\varphi_{-\infty} p_{\text {a }}$ ist der maximale Auslenkungswinkel der ersten und $\tau_{a}$ der der zweiten Schwingung. Das Verhältnis der beiden Winkel $\left(\varphi_{a} / p-\varphi_{a}\right)$ ist im allgemeinen klein. Sie verhalten sich grössenordnungsmässig umgekehrt wie die Quadrate der Frequenzen. Daher tritt die hochfrequente Schwingung in den Registrierungen nicht in Erscheinung, zumindest nicht nennenswert.

4. Losung der inhomogenen Differentialgleichungen. Die erzwungenen Schwingungen. - Als Ausgangsgleichungen dienen wieder die Gl. [5a] und [5b]. Die sinusförmige Bewegung des Bodens setzen wir in der Form an:

$$
\eta=F \cos \omega t
$$

Die Differentialgleichungen lauten somit:

$$
\begin{array}{r}
\left(K_{0}+M a^{2}\right) \frac{d^{2} \varphi}{d \iota^{2}}+2 \varrho s^{2} \frac{d \varphi}{d t}+\left(\frac{B}{l}+c r^{2}\right) q+M a \frac{d^{2} y^{\prime}}{d \iota^{2}}+2 \varrho s \frac{d y^{\prime}}{d t}+ \\
-c r y^{\prime}=M a F \omega^{2} \cos \omega t ; \\
M a \frac{d^{2} \varphi}{d \iota^{2}}+2 y \frac{d \varphi}{d t}+c r \varphi+M \frac{d^{2} y}{d t^{2}}+2 \varrho \frac{d y^{\prime}}{d t}+\left(\frac{12 B}{l^{3}}+\right. \\
+c) y^{\prime}=M F \omega^{2} \cos \omega t ;
\end{array}
$$

Die Lösungen setzen wir wie üblich in der Form an:

$$
\varphi=p_{1} \cos \omega t+q_{1} \sin \omega t ; y^{\prime}=p_{2} \cos \omega t+q_{2} \sin \omega t ;[18]
$$

Nach Bildung der ersten und zweiten Differentialquotienten von Gl. [18] und Einsetzen derselben in Gl. [17] erhält man für $p_{1}, q_{1}, p_{2}$ und $q_{2}$ aus der Bedingung, dass in Gl. [17a] und [17b] die Koeffizienten der cos- wie die der sin-Glieder verschwinden müssen, folgende Bestimmungsgleichungen:

$$
\begin{gathered}
\left(\frac{B}{l}+c r^{2}-\left(K_{0}+M a^{2}\right) \omega^{2}\right) p_{1}+2 \varrho s^{2} \omega q_{1}+\left(c r-M a \omega^{2}\right) p_{2}+2 \varrho s \omega q_{2}=M a F \omega^{2} \\
-2 \varrho s^{2} \omega p_{1}+\left(\frac{R}{i}+c r^{2}-\left(K_{0}+M a^{2}\right) \omega^{2}\right) q_{1}-2 \varrho s \omega p_{2}+\left(c r-M a \omega^{2}\right) q_{2}=0 \\
\left(c r-M a \omega^{2}\right) p_{1}+2 \varrho s \omega q_{1}+\left(\frac{12 D}{l^{3}}+c-M \omega^{2}\right) p_{2}+2 \varrho q_{2}=M F_{0^{2}}
\end{gathered}
$$




$$
-2 \varrho s \omega p_{1}+\left(c r-M a \omega^{2}\right) q_{1}-2 ! p_{2}+\left(\frac{12 B}{l^{3}}+c-M \omega^{2}\right) q_{2}=0
$$

Die Auflösung dieses Gleichungssystems nach den Grössen $p_{1}$, $p_{2}, q_{1}$ und $q$ ? macht bei der speziellen Gestalt der Koeffizienten keine grosse Mühe. Zweckmässigerweise führt man folgende Abkürzungen ein, wobei bereits die früher besprochenen Vernachlässigungen gemacbt werden:

$$
\begin{aligned}
R & =\frac{B / l+c r^{2}}{K_{0}+M a^{2}} \frac{1}{\omega^{2}}-1+\frac{l^{3} M K_{0}}{12 B\left(K_{0}+M a^{2}\right)} \omega^{2}+\frac{c l^{3}}{12 B}\left(\frac{B / l}{K_{0}+M a^{2}} \frac{1}{\omega^{2}}-1\right) \\
& -\frac{l^{3} M\left(B / l+c r^{2}-2 c r a\right)}{12 B\left(K_{0}+M a^{2}\right)} \cong \frac{0_{i}^{2}}{\omega^{2}}-1+\frac{\omega^{2}}{\omega_{2}^{2}} ; \\
S & =\frac{2 \varrho s^{2}}{K_{0}+M a^{2}}\left(1+\frac{l^{2}}{12 s^{2}}+\frac{c l^{3}}{12 B} \frac{(r-s)^{2}}{s^{2}}-\frac{l^{3} \omega 2 \varrho\left(K_{0}+M(a-s)^{2}\right)}{12 B\left(K_{0}+M a^{2}\right)}\right. \\
& =\frac{2 \delta_{1}}{\omega}-\frac{2\left(\delta_{1}+\delta_{2}\right) \omega}{\omega_{n^{2}}^{2}} ; \\
U_{1} & =\frac{2 \varrho(a-s) l^{3}}{12 B\left(K_{0}+M a^{2}\right) \omega}=\frac{2 \delta_{1}(a-s) l^{3}}{12 B s^{2} \omega} \cong 0 ; \\
V_{1} & =\frac{a}{K_{0}+M a^{2}} \frac{1}{\omega^{2}}\left(1+\frac{c l^{3}}{12 B}\left(1-\frac{r}{a}\right)\right) \cong \frac{a}{K_{0}+M a^{2}} \frac{1}{\omega^{2}} ; \\
U_{2} & =\frac{2 \varrho s(a-s) l^{3}}{12 B\left(K_{0}+M a^{2}\right) \omega}=\frac{2 \delta_{1}(a-s) l^{3}}{12 B s \omega} \cong 0 ; \\
V_{2} & =\frac{l^{3}}{12 B\left(K_{0}+M a^{2}\right) \omega^{2}}\left(B / l+c r\left(r-x^{2}-K_{0} \omega^{2}\right) \cong 0 ;\right. \\
L & =a\left(1+\frac{K_{n}}{M a^{2}} j ;\right.
\end{aligned}
$$

Damit lassen sicb die Ausdrïcke für $p_{1}, q_{1}, p_{2}$ und $q_{2}$ einfach darstellen.

$$
\begin{aligned}
& p_{1}=\frac{U_{1} S+V_{1} R}{R^{2}+S^{2}} M F\left(\omega^{2}=\frac{F}{L} \frac{R}{R^{2}+S^{2}} ;\right. \\
& q_{1}=\frac{V_{1} S-U_{1} R}{R^{2}+S^{2}} M F \omega^{2}=\frac{F}{L}-\frac{S}{R^{2}+S^{2}} ; \\
& p_{2}=\frac{-I I_{2}+V_{2} R}{R^{2}+S^{2}} M F \omega^{2} \cong 0 ; q_{2}=\frac{V_{2} S+U_{2} R}{R^{2}+S^{2}} M F \omega^{2} \cong 0 ;
\end{aligned}
$$


Setzt man nun diese Grössen in Gl. [18] ein, so ergilbt sich:

$$
\varphi=\frac{F}{L} \mathrm{~V}_{\mathrm{d}} \cos (\omega t-\gamma) ; y^{\prime} \cong 0
$$

wobei $\mathrm{V}_{\mathrm{d}}=1 / \sqrt{R^{2}+S^{2}}$; und $\operatorname{tg} \gamma=S / R$;

Für die vom Seismographen aufgeschriebene Kurve erhalten wir folgende Gleichung:

$$
y_{J}=J \varphi+y^{\prime}=\frac{F}{L} J \mathrm{~V}_{\mathrm{d}} \cos (\omega t-\gamma) ;
$$

Damit ist die inhomogene Differentialgleichung auch völlig gelöst. Im Falle sinusförmiger erzwungener Schwingungen bleibt bei einem Seismographen mit Blattfederaufhängung die Drehaclsse der schnelleren Koppelschwingung in Raume in Ruhe, zumindest ist ihre Bewegung so klein, dass sie praktisch keine Rolle spielt. Die statische Vergrösserung eines Apparates $V_{\mathrm{s}}$ ist das Verhältnis der Indikatorlänge $J$ (Alsstand zwischen Blattfedermitte und Ende des Indikators) zum Alsstand der Drehachsen der beiden Koppelschwingungen L. Die dynamische Vergrösserung $V_{d}$ zeigt das Verhalten eines gekoppelten Schwingungsgebildes mit zwei entfernt voneinander liegenden Koppelfrequenzen. Bei diesen treten Resonanzstellen auf. Bei kleinen Frequenzen beginnt die Resonanzkurve mit dem Wert $O$ und steigt zur ersten Resonanzstelle an, die allerdings durch die Dämpfungsvorrichtung unterdrückt werden kann. Zwischen den beiden Resonanzstellen erreicht die dynamische Vergrösserung den Wert 1. Die zweite Resonanz bei $v_{2}$ wird durch die üblichen, für die hohen Frequenzen zu schwach arbeitenden Dämpfungen kaum beeinflusst. Nach noch höheren Frequenzen hin sinkt die Vergrösserung der Seismographen wieder auf den Wert $O$ als. In Fig. 2 ist die Resonanzkurve und die Phasenverschiebungskurve für ein Beispiel eingetragen. Die beiden Koppelfrequenzen wurden zu $v_{1}=1 \mathrm{~Hz}$ und $v_{2}=40 \mathrm{~Hz}$ angenommen. Die Kurve $(a)$ stellt den dämpfungsfreien Fall $(\varrho=O)$ dar. Bei der Kurve $(b)$ wurde aperiodische Dampfung für $v_{1}$ angenonmen $\left(\delta_{1}=\omega_{1}\right)$ und $\delta_{2}=2 \delta_{1}$ gesetzt. Die Phasen werden durch jede Resonanzstelle un $\pi$ verschoben.

5. Zusammenfassung und Folgerungen. - Es soll hier nochmals kurz der Inhalt der vorstehenden Abschnitte zusammengefasst werden, un daran die Folgerungen für den Apparatebau albzuleiten. Wir haben gesehen, dass ein einseitig eingespannter Blattfederseismograph 
kein einfaches Schwingungsgebilde, sondern ein aus zwei gekoppelten Eigenfrequenzen bestehendes System ist. Die eine Eigenschwingung ist eine Drehschwingung der Masse um einen Punkt, der um die Hälfte
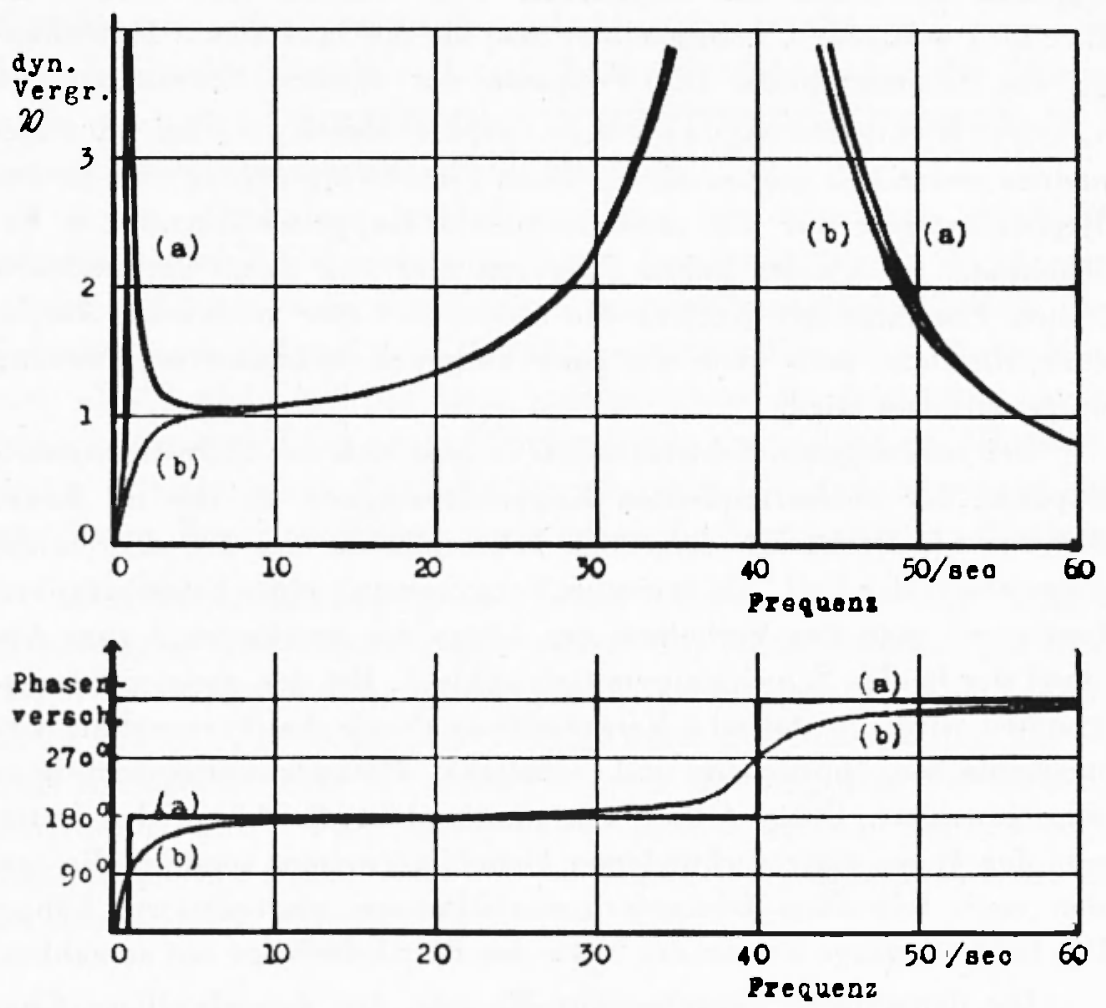

Fig. 2 - Die Ablïngigkeit der dynamischen Vergrösserung und der Phasenverschiebung eines Blattfederseismographen bei erzwungenen Sclwingungen von der Frequenz der Bodenbewegung. Der Bere.hnung wurden für die beiden Koppelfrequen. zen des Apparates die Werte $v_{1}=1 / \mathrm{s}$ und $v_{2}=40 / \mathrm{s}$ zu Grunde gelegt. Kurve $(a)$ gilt für den dämpfungsfreien Fall $(Q=0)$. Kurve $(b)$ gilt für den Fall aperiodischer Dampfung fiir $v_{1}\left(\delta_{1}=\omega_{1} ; \delta_{2}=2(1)_{1}\right)$.

der Blattfederlänge vom Einklemmpunkt am Stativ entfernt ist. Die Frequenz beträgt $v_{\varphi}=\sqrt{\left(B / l+c r^{2}\right) / 4} \pi^{2}\left(K_{0}+M a^{2}\right)$. Die zweite Eigenschwingung ist eine Translationsschwingung des Systems. Hier ist die Frequenz $v_{\mathrm{T}}=\sqrt{12 B / 4 \pi^{2} l^{3} M}$. Diese beiden Schwingungen sind stark miteinander gekoppelt. Es wird daher nur in Ausnahmefällen möglich sein, eine von heiden rein zu erhalten. Durch die Kopplung entstehen aus den Eigenfrequenzen zwei Koppelschwingungen, die sich alle beide als Drehschwingungen erweisen. Die erste unterscheidet sich von der Dreheigenschwingung nur um kleine, vernachlässigbare Grös- 
sen. Die Drehachsen und die Frequenzen fallen praktisch zusammen. Die zweite Koppelschwingung hat ihre Drehachse im Schwingungsmittelpunkt der ersten und ungekehrt. Der Abstand der beiden ist $L=a\left(1+K_{0} / \boldsymbol{M a}^{2}\right)$. $L$ bezeichnet man als die äquivalente Pendellänge des Seismographen. Die Frequenz der zweiten Schwingung ist $v_{2}=\sqrt{12 B\left(1+M a^{-} / K_{0}\right) / 4 \pi^{2} b^{3} M}=v_{1} \sqrt{1+M a^{2} / K_{0}} \cdot v_{2}$ ist im allgemeinen wesentlich grosser als $v_{1}$. Beim Einschwingvorgang tritt in den Registrierungen nur die niederfrequente Koppelschwingung in Erscheinung. Infolge der hohen Frequenz und dem damit verbundenen hohen Energiegehalt erreicht die zweite nie eine merkliche Amplitude, die dann auch noch mit einer kleineren Indikatorvergrösserung aufgeschrieben wird.

Bei erzwungenen Schwingungen erweist sich der Schwingungsmittelpunkt der niederfrequenten Koppelschwingung als die im Raum feststehende Drehachse derselben. Jedenfalls ist dies bei den hohen Frequenzen der Fall. Als statische Vergrösserung eines Seismographen bezeichnet man das Verhaltnis der Länge des Indikators $\boldsymbol{J}$ zum $\mathrm{Ab}$ stand der beiden Sclıwingungsmittelpunkte $L$. Bei den meisten Seismographen wird die statische Vergrosserung durch die Verwendung von mechanischen, optischen und sonstigen Übersetzungsvorrichtungen sehr gesteigert. Unter $J$ darf man dann nicht die Länge des ersten mit der Masse starr verbundenen Hebels verstehen, sondern die mit den noch folgenden Übersetzungsverhältnissen multiplizierte Länge. Die Indikatorlänge ist von der Mitte der Blattfederlänge aus zu zählen.

Die dynamische Vergrösserung $V_{d}$ zeigt den doppelwelligen Charakter, wie er von der Theorie der Koppelschwingungen ber bekannt ist. Wahrend die niederfrequente Resonanzstelle durch die Dämp. fungseinrichtung weggedämpft werden kann, ist dies bei der zweiten nicht möglich. Die Phasenverschiebung wächst von den niedrigen zu den hohen Frequenzen hin von $O$ bis $2 \pi$.

Fir den Bau von Seismographen ergeben sich daraus wichtige Folgerungen. 1. Un eine möglichst wahrheitsgetreue Aufzeichnung der Bodenbewegungen zu erhalten, sind die beiden Koppelfrequenzen möglichst so zu legen, dass die erste an unteren Ende des Bereiches liegt, in welchen die hauptsächlichen Frequenzen der aufzuzeichnenden Bodenbewegungen fallen, während die zweite weit nach oben zu schieben ist. Da man die Apparate nicht unnötig schwer machen will, wird die träge Masse nicht grosser gewählt als es notwendig ist. Thre Grösse, bezw. die Grösse der Trägheitskräfte, muss ausrei- 
chen, um die am System angreifenden Reibungskräfte zu überwinden. Die Frequenz der ersten Koppelschwingung kann man durch Wahl der Direktionskräfte von Blatt- und Spiralfeder geeignet legen. Die Frequenz der zweiten Koppelschwingung wird gross, wenn die freie Länge der Blattfeder $l$ und $R$, der Trägheitsradius der Masse, klein wird. $R$ ist definiert durch $R=\sqrt{K_{0} / M}$. $\ldots$ wird aber durch $t$ wesentlich stärker beeinflusst als durch $R$.

2. Im allgemeinen möchte man ein Instrument mit möglichst grosser statischer Vergrösserung haben. Dies erreicht man durch eine grosse Indikatorlänge $J$ und einen möglichst kleinen Ahstand der Schwingungsmittelpunkte $L$. Durch die notwendige träge Masse lässt sich aber $L$ nicht beliebig klein machen. Unter der Bedingung gleich grosser Masse wird bei quaderförmiger Gestalt derselhen ein Minimum für $L$ erreicht, wenn die Ilöhe des Quaders 1,15 mal grösser ist als die Breite. Bei zylindrischer Masse liegen die Verhältnisse ungünstiger.

6. Blattfederseismographen anderer Bauart. - Die vorstehenden Rechnungen und Úberlegungen gelten für Blattfederseismographen, deren Konstruktion dem Schema der Fig. 1 entsprechen. Die Endformeln ändern sich jedoch sehr wenig, wenn die Konstruktion anders durchgeführt ist. Dies soll am wichtigsten Beispiel, dem Vertikalseismographen nach A ngenheiste $r$ gezeigt werden. Fig. 3 stellt das Schema der Bauart dar. Für das Moment der Dämpfungskraft ist zil setzen:

$$
M_{\mathrm{d}}=2 \varrho s^{2} \frac{d \varphi}{d t}
$$

Danach fallen in den Gleichungen [4] und [5] die Glieder mit $d y_{1} / d t$ bezw. $d y^{\prime} / d t$ weg. Die Ausdrücke für die Frequenzen ändern sich dadurch nicht. Geringfügige Abweichungen treten nur bei den Ausdrücken für die Dämpfungen auf. Während sich derjenige für die niedrige Frequenz formal nicht ändert, vereinfacht sich der für die hohere. Sie lauten:

$$
\delta_{1}=\frac{\rho s^{2}}{K_{0}+M a^{-2}} ; \delta_{2}=\frac{s^{2}}{K_{n}}-\frac{\varrho s^{2}}{K_{0}+M a^{2}}=\frac{\rho s^{2}}{K_{0}+M a^{2}} \frac{M a^{2}}{K_{0}} ;
$$

Auch die Formeln für die Resonanzkurve bleiben hestehen.

Durch eine andersartige Anordnung der Einzelteile der Seismographen wird ihr Verhalten nur wenig beeinflusst. Es ist daher nicht nötig, alle Möglichkeiten einzeln durchzudiskutieren, zumal im zwei- 
ten, im experimentellen. Teil der Arbeit auf eine weitere Bauart näher eingegangen wird.

II. Teil. Experimentelle Untersuciiungen an BlattfederseismograPHEN.

Die Anregungen zu den obigen theoretischen Darlegungen ist durch experimentelle Untersuchungen von Blattfederseismographen

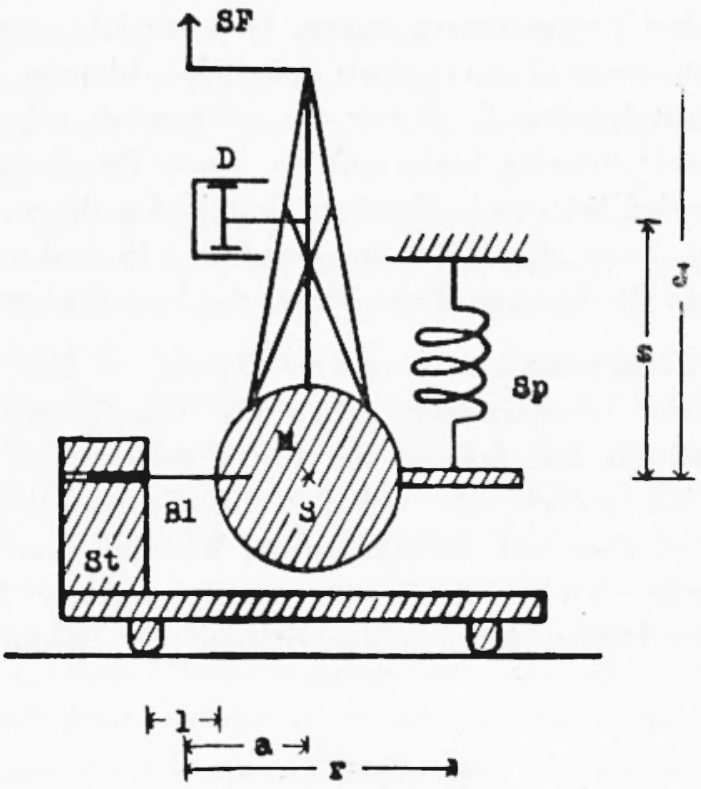

Fig. 3 - Schema des Vertikalseismographen nach Ange nheister. St $=$ Stativ mit Klemmvorrichtung fiir die Blattfeder. $M=$ Masse des Seismographen. $S=$ deren Schwerpunkt. $S p=$ Spiralfeder. $D=$ Dampfung. $S F=$ Schreibfeder bezw. Ende des an der Masse befestigten starren Hebels. $B l=$ Blattfeder. $l=$ freie Länge der Blattfeder. $a=$ Abstand Blattfedermitte-Schwerpunkt. $r=$ Abstand Blattfedermitte-Angriffspunkt der Spiralfeder. $s=$ Abstand Blattfeder-Angriffspunkt der Dampfung. $J=$ Abstand Blattfeder-Sclireibfeder.

auf Schütteltischen gegeben worden. In diesem Teil der Abhandlung soll gezeigt werden, wie weit die theoretischen und die experimentellen Ergebnisse übereinstimmen und welche Nebenumstände noch einen grossen Einfluss auf die Arbeitsweise eines solchen Apparates haben können.

1. Untersuchungen am transportablen Wiechertschen Horizontalseismographen. - Es handelt sich bierbei um einen der ersten trans- 
portablen Seismographen (*). Der schematische Aufbau des Gerätes ist in Fig. 4 dargestellt. Die Masse ist an zwei Kreuzfederpaaren hefestigt. Ihr Schwerpunkt liegt senkrecbt iiber der Verbindungslinie der Kreuzungspunkte der Federn. Die Direktionkraft der Schwingungen dieses Systems setzt sich aus zwei Komponenten zusammen. Die eine wird von den Blattfedern geliefert, während die andere von der Wirkung der Schwerkraft auf die Masse des Seismographen geliefert wird. In der normalen Aufstellung, wie sie Fig. 4 zeigt, wirken beide Kräfte einander entgegen. Dreht man das Gerät um, so dass die Masse an den Blattfedern hänğt, dann addieren sich die Kräfte. Bei diesem
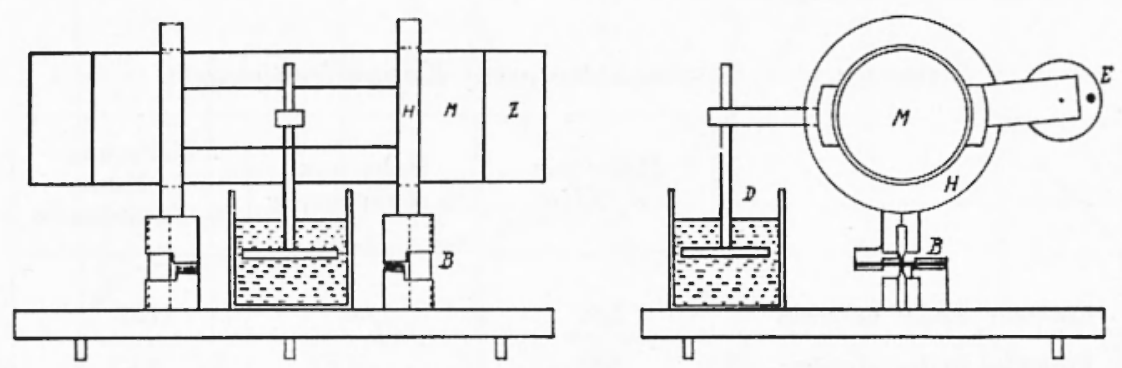

Fig. 4 - Schema des Horizontalseismographen nach Wiechert im Seiten- und Aufriss. $M=$ Masse des Seismographen. $H=$ Halterung derselben. $B=$ Blattfederkreuz. $Z=$ Zusatzmasse aus Blei. $D=$ Dïmpfungsvorrichtung (öldampfung). $E=$ Exzenterrädchen.

Apparat lässt ich die Masse leicht veründern. Man kann auf diese Weise den Einfluss der einzelnen Elemente auf die erste Koppelfrequenz prüfen. Die Resonanzkurve und die Lage der zweiten Koppelschwingung im Frequenzhand wurde nach der von $R$. Kö h l e r (5) angegehenen Methode mit Hilfe eines Schwungrädchens untersucht.

a) Die erste Koppelfrequenz. Die Formel für die erste Koppelfrequenz lautet:

$$
v=\sqrt{\frac{B / l+M g a}{K_{0}+M a^{2}}}
$$

$\mathrm{Das}$ positive Vorzeichen im Zähler des Ausdruckes gilt für hängende, das negative für aufrechtstehende Masse. Durch eine Änderung

(*) Eine gute Abbildung des Apparates ist auf dem Bild von E. WIEchrrT zu sehen. Zeitschr. f. Geophysik, Bd. IV (1928) S. 49. 
der Masse wird sowohl der Zähler wie der Nenner unter der Wurzel beeinflusst. Bei diesem Gerät kann die zylinderförmige Masse leicht aus ilırer Halterung entfernt werden. Ausserdem kann sie durch das Ansclirauben von Zusatzmassen aus Blei vergrössert werden. Die Gewichte und Trägheitsmomente der Einzelmassen bezogen auf den Kreuzungspunkt der Blattfederpaare sind bestimmt worden. Die Eigenperioden des Schwingungssystems sind bei verscbiedenen Kombinationen der Einzelmassen in aufrechtstehender wie in bängender Lage gemessen worden. Bei den meisten Versucben sind die waagrecht liegenden Federn der Kreuzfederpaare abgenommen worden. Tabelle 1 bringt die Messergebnisse.

Tabelle 1 - Beobachtete erste Koppelfrequenzen

\begin{tabular}{|l|c|c|c|}
\hline & $\begin{array}{c}\text { Halterung } \\
+ \text { Masse }\end{array}$ & $\begin{array}{c}\text { Halterung } \\
+ \text { Zusatzmasse }\end{array}$ & $\begin{array}{c}\text { Halterung } \\
\text { + Masse } \\
+ \text { Zusatzmasse }\end{array}$ \\
\hline Einfache Feder aufrecht & 1,62 & 2,99 & 1,25 \\
Einfache Feder abwarts & 2,87 & - & 2,67 \\
Kreuzfeder & - & - & 2,63 \\
\hline
\end{tabular}

An Hand der Dimensionen der Einzelteile des Schwingungssystems lassen sich nach obiger Formel die einzelnen Frequenzen auch berechnen. Beim Vergleich der berechneten Werte mit den gemessenen zeigt sich, dass sich bei einer Änderung der Masse die Frequenzen so ändern, wie es die Theorie erfordert. Die Versuche ergaben darïber linaus weiterhin, dass die Direktionskraft der Blattfedern nicht, wie zunächst zu erwarten wäre, konstant bleibt, sondern eine gewisse Abhängigkeit von der Vorbelastung zeigt. Bei aufrechtstehendem System wird durch die Druckbelastung der rund $5 \mathrm{~kg}$ schweren Masse die Direktionskraft der vertikalen Blattfedern, deren Quer. schnitte aus Fig. 4 zu ersehen ist, um 8,6\% vermindert. Bei luängender Masse ist sie um denselben Betrag grösser. Mit abnehmender Grösse der Masse wird der Prozentsatz geringer. Genauere Angaben über die Gesetzmässigkeit dieser Erscheinung lassen sich aus den wenigen Werten nicht ableiten. Bei Kreuzfedern wird die Direktionskraft der Sclıwingungen melır als vervierfacht, obwohl die Biegesteifigkeit der waagrechten Federn geringer ist als die der senkrechten.

b) Die zweite Koppelfrequenz und die Resonanzkurve. Es wur- 

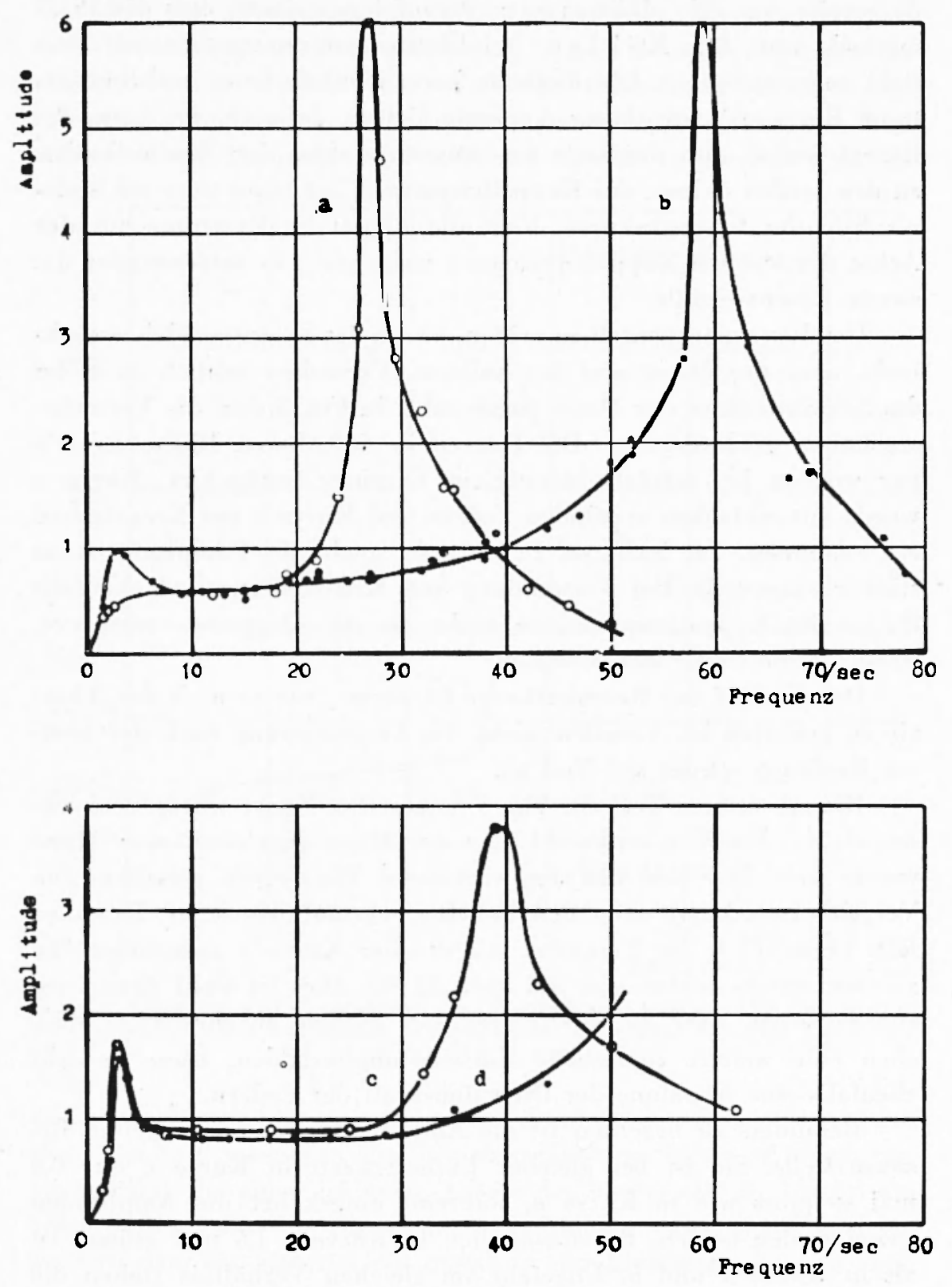

Fig. 5 - Resonanzkurven des Horizontalseismographen nach W i e c $\mathrm{h}$ e $\mathrm{r}$ aufgenommen mit der Schwungradmethode. Die Anplituden sind in willkürlichen Einheiten aufgetragen. Bei Kurve a und $b$ befand sich das Schwungrad seitlich vom Schwerpunkt der Masse, bei Kurve $c$ und $d$ senkrecht darüber. Kurve $a$ und $c$ wurden bei einfachen, vertikalen Blattfedern, Kurve $b$ und $d$ bei Kreuzfedern aufgenommen. 
de bereits von M. Rössi ge $r$ darauf hingewiesen, dass die Prüfmethode von $R$. Köhler bei Blattfederseismographen mit Vorsicht anzuwenden ist. Die Methode kann nämlich keine wahrheitsgetreue Resonanzkurve dieser Apparate liefern. Je nach der Lage der Exzenterachse, also der Lage des Angriffspunktes der Wecliselkräfte, zu den beiden Achsen der Koppelfrequenzen bekommt man ein anderes Bild der Resonanzkurve. Fallt die Achse des Exzenters mit der Achse der zweiten Koppelschwingung zusammen, so verschwindet die zweite Resonanzstelle.

Um dies experimentell zu prüfen, wurde das Exzenterrädchen senkrecht über der Masse und bei anderen Versuchen seitlich in Höhe des Scliwerpunktes der Masse angebracht. In Fig. 5 sind die Versuchsergebnisse wiedergegeben. Die Kurven in der oberen IHälfte der Figur wurden bei seitlich befestigtem Exzenter beobachtet. Kurve a wurde mit einfachen vertikalen Federn und Kurve $b$ mit Kreuzfeilcrn aufgenommen. Im letzteren Fall wurde auch die Dämpfung etwas stärker eingestellt. Bei Verwendung von Kreuzfedern wird ebenfalls die zweite Koppelfrequenz um melır als das Doppelte vergrössert, sie steigt von $27 \mathrm{~Hz}$ anf $60 \mathrm{~Hz}$ an.

Der Verlauf der Resonanzkurve ist derart, wie er nach der Theorie zu erwarten ist. Vorallem sinkt die Vergrösserung nach der zweiten Resonanz wieder auf Null ab.

Die im unteren Teil der Fig. 5 dargestellte Kurve wurde beobachtet, als das Rädchen senkrecht ïber der Masse angchracht war. Diese wurde auch hier nur von den vertikalen Blattfedern gehalten. Ihie Vergrösserungskurve zeigt den üblichen Verlauf. Die erste Resonanz fallt hinsichtlich der Frequenz mit der der Kurve a zusammen. Die zweiten unterscheiden sich um etwa $22 \mathrm{~Hz}$. Dies ist wohl darauf zurückzufülıren, dass die Blattfedern bei seitlich angebrachtem Rädchen eine weitere zusätzliche Vorbelastung erleiden. Diese bewirkt ebenfalls eine Abnahme der Direktionskraft der Federn.

Besonders zu beachten ist die Amplitudengrösse der zweiten Resonanzstelle. Sie ist bei gleicher Exzenterkraft in Kurve $c$ nur 0,6 mal so gross wie in Kurve $a$, während umgekehrt die Amplituden zwischen den beiden Resonanzstellen in Kurve $c$ 1,6 mal grösser ist als in Kurve $a$ und $b$. Ungefahr im gleichen Verhältnis stehen die Abstände der Exzenterachse von den Achsen der Koppelf requenzen.

2. Untersuchungen an Vertikalseismographen nach Angenheister. Der Einfluss der Einklemmung der Blattfedern auf das 
Verhalten der Seismographen. - In einer weiteren Versuchsserie wurden Vertikalseismographen der Bauart Angen h e is te r näher untersucht. Dahei ergahen sich erhehliche Differenzen zwischen den theoretisch berechneten und den durch Experimente gefundenen Werten der Koppelfrequenzen. Der Grund hierfür ist in der Einklem-

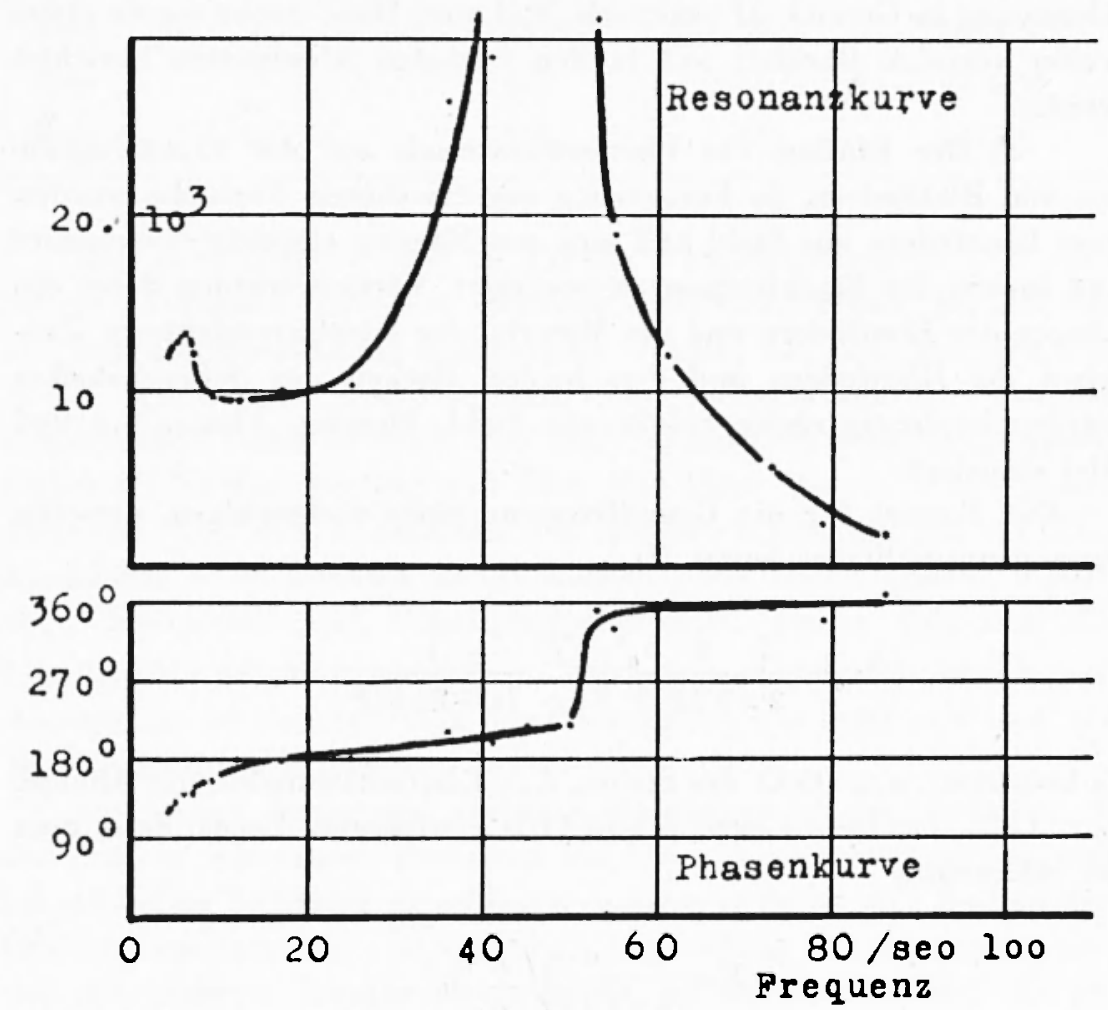

Fig. 6 - Resonanz- und Phasenkurve des Vertikalseismographen Z12. Die Blattfedern waren zwischen ausgegliihte Messingbacken geklemmt. Die freie Länge der Blattedern betrug $0,02 \mathrm{~cm}$.

mung der Blattfedern zu suchen. Der Einfluss der Einklemmung auf die Eigenfrequenzen von einseitig eingespannten Stäben ist schon lange hekannt. In einer früheren Arheit des Verfassers $\left({ }^{6}\right)$ konnte gezeigt werden, dass im allgemeinen die Einklemmung nicht ideal ist, sondern dass das Material des Stahes in der Einspannstelle und auch das der Klemmhacken auf die Schwingungen mit einwirkt. Um diesem Effekt in den Formeln zur Berechnung der Eigenfrequenzen Rechnung zu tragen, muss man in ihnen statt der freien Stahlänge $l$ seine wirksame einsetzen. Die wirksame Länge ist bei Schwingun- 
gen um den Betrag $2 \Delta l$ grösser als die freie. Bei statischer Belastung ist sie nur un das Stiick $\Delta l$ grösser. $\Delta l$ hängt vom Material des Stabes und von dem der Klemmvorrichtung ab. Bei einem in Stahlbakken eingespannten Holzstab wurde bei freien Stablängen von 0,75$-1,00 \mathrm{~m} \Delta l$ zu etwa $1 \%$ derselben bestimmt, während bei einer Klemmung in Gummi $\Delta l$ praktisch Null war. Diese Sache wurde etwas weiter verfolgt. Darïber soll in den nächsten Abschnitten berichtet werden.

a) Der Einfluss des Einspannmaterials auf die Eigenfrequenzen von Blattfedern. In Fortsetzung der erwähnten Versuche wurden zwei Blattfedern aus Stahl und eine aus Messing einseitig eingespannt und jeweils die Eigenfrequenzen bestimmt. Variiert wurden dabei die Längen der Blattfedern und das Material der Klemmvorrichtung. Zwischen die Blattfedern und den beiden Backen des Schraubstockes wurden beidseitig ebene Stücke aus Stahl, Messing, Aluminium und Blei eingelegt.

Die Formel für die Grundfrequenz eines rechteckigen, einseitig eingespannten Stabes lautet ( ${ }^{7}$ ).

$$
v_{0}=\frac{1}{2 \pi} \frac{a}{\sqrt{12}} \frac{\beta_{0}^{2}}{l^{2}} \sqrt{\frac{E}{\varrho}} \frac{1}{(1+2 \Delta l / l)^{2}} ;
$$

Es bedeuten : $a=$ Dicke des Stabes, $E=$ Elastizitätsmodul, $\varrho=$ Dichte, $\beta_{0}=1,875, l=$ freie Länge, $l(1+2 \Delta \bar{l} / l)=$ wirksame Länge. Setzt man zur Abkiirzung

$$
K=\frac{1}{2 \pi} \frac{a}{\sqrt{12}} 1 / \sqrt{\frac{E}{\varrho}} \beta_{0}^{2}
$$

so erhält man für $\Delta l$ aus

$$
v_{0}=K\left(\frac{1}{l(1+2 \Delta l / l)}\right)^{2}: \Delta l=\frac{l}{2}\left(1 \sqrt{\frac{K}{v_{0} l^{2}}}-1\right) ;
$$

$K$ kann man berechnen, wenn man $E$ genügend genau kennt. Die Bestimmung von $E$ war Gegenstand weiterer Messungen. Die Blattfedern wurden dabei an den Enden auf zwei Schneiden aufgelegt und zu Biegeschwingungen angeregt. Die Frequenzen derselben wurden gemessen und daraus $E$ und dann $K$ berechnet. In Tabelle 2 sind die technischen Daten der Blattfedern angegeben. 
TABelle 2

Technische Daten der Blattfedern

\begin{tabular}{|c|c|c|c|c|c|c|c|c|}
\hline & Blattfede & a & us & & & $\begin{array}{c}\text { Dicke a } \\
\text { cm }\end{array}$ & $\begin{array}{c}\text { Breite } b \\
\mathrm{~cm}\end{array}$ & $\underset{\mathrm{cm} \mathbf{2}^{2} / \mathrm{s}}{\mathrm{K}}$ \\
\hline Stahl & . & & - & & . & 0,04 & 2,5 & $3,05.10^{3}$ \\
\hline Stahl & - & ${ }^{\circ}$ & . & & . & 0,01 & 1,5 & $0,855.10^{3}$ \\
\hline Messing & . . & . & . & & . & 0,10 & 1,95 & $5,67.10^{3}$ \\
\hline
\end{tabular}

Die Messergebnisse sind in Tabelle 3 zusammengestellt. Auf eine nähere Diskussion derselben soll hier verzichtet werden. Es soll nur auf folgende Feststellung hingewiesen werden, die auch mit den früheren Erfahrungen ïbereinstimmt. Als Klemmbacken für einseitig eingespannte Stäbe ist Stahl und auch Aluminium schlecht geeignet. Besser ist hierfür Messing und Blei. Für diese Metalle wird $\Delta l$ am kleinsten. Von den Konstanten des Klemmaterials, die auf die Einklemmung einen Einfluss haben könnten, wie Härte, Dichte, Elastizität, Kompressibilität, Schallgeschwindigkeit, ändert sich nur die Schallgeschwindigkeit $v=\sqrt{E / \varphi}$ ähnlich wie $\Delta l$. Für Stahl und Aluminium ist sie $5100 \mathrm{~m} / \mathrm{s}$, für Messing ist $v=3400 \mathrm{~m} / \mathrm{s}$ und für Blei $v=1300 \mathrm{~m} / \mathrm{s}$.

Auf Grund dieser und früherer Messungen ist der Verfasser zu der Ansicht gekommen, dass man bei freien Eigensclıwingungen und bei statischer Belastung einseitig eingespannter Stäbe und Balken der idealen Einklemmung $(\Delta l=O)$ bei Verwendung von Einspannmaterial mit geringer Schallgeschwindigkeit am nächsten kommt. Es genügt hierbei, wenn man zwischen Stah und Klemmvorrichtung, Schrauhstock, usw., Stücke von der Dicke weniger Millimeter aus einem solchen Stoff einlegt.

$b$. Der Einfluss der Klemmung auf die Koppelfrequenzen und die Resonanzkurve von Blattfederseismographen. Nach den obigen Beobachtungsergebnissen ist zu vermuten, dass die Art der Einklemmung der Blattfedern von Seismographen einen Einfluss auf deren Verhalten ausüben wird. Die Vertikalseismographen Z10, Z11 und Z12 des Geophysikalischen Institutes Göttingen dienten zur nähren Untersuchung dieses Einflusses. Die drei Apparate sind von der gleichen Bauart und nach den Angaben von Angenheister entwickelt worden. Sie wurden auf dem Vertikalschütteltisch des Göttin- 


\section{Tabfle 3}

Der Einspannwert $\Delta l$ in Abhängigkeit von der Stablänge, dem Stabmaterial und dem Einspannmaterial berechnet aus Schwingungsbeobachtungen.

\begin{tabular}{|c|c|c|c|c|c|c|c|c|}
\hline Backen aus & \multicolumn{2}{|c|}{ Stahlı } & \multicolumn{2}{|c|}{ Alluminium } & \multicolumn{2}{|c|}{ Messing } & \multicolumn{2}{|c|}{ Blei } \\
\hline Fider nus & Stahl & Messing & Stahl & Messing & Stnhl & Mersing & Stalıl & Messing \\
\hline $\begin{array}{l}\text { Stablange } \\
1 \text { (cm) }\end{array}$ & $\begin{array}{c}\Delta l \\
(\mathbf{c m})\end{array}$ & $\begin{array}{l}\Delta l \\
(\mathrm{~cm})\end{array}$ & $\begin{array}{l}\Delta \mathrm{l} \\
(\mathrm{cm})\end{array}$ & $\underset{(\mathrm{cm})}{\Delta \mathrm{l}}$ & $\underset{(\mathrm{em})}{\Delta \mathrm{l}}$ & $\underset{(\mathrm{cm})}{\Delta \mathrm{l}}$ & $\underset{(\mathrm{cm})}{\Delta \mathrm{l}}$ & $\underset{(\mathrm{cm})}{\Delta \mathrm{l}}$ \\
\hline 4 & 0,2 & - & 0,2 & - & 0,0 & - & 0,0 & - \\
\hline 6 & 0,2 & -- & 0,1 & - & 0,1 & - & 0,0 & - \\
\hline 8 & 0,2 & 0.3 & - & - & 0,0 & - & 0,0 & - \\
\hline 10 & 0,4 & 0,4 & 0,1 & 0,2 & 0,0 & 0,0 & $0^{i} 0$ & 0.0 \\
\hline 12 & 0,4 & - & - & - & 0,0 & - & - & - \\
\hline 14 & 0,5 & 0,4 & 0,2 & - & 0,0 & - & 0,1 & - \\
\hline 16 & 0,5 & - & - & - & 0,0 & - & - & - \\
\hline 18 & 0,6 & 0,4 & 0,2 & 0,3 & 0,0 & 0,1 & 0.0 & 0,1 \\
\hline 20 & 0,6 & 0,3 & - & - & 0,0 & - & - & - \\
\hline 22 & 0,5 & $\therefore$ & 0,2 & - & 0,0 & - & 0,0 & - \\
\hline 24 & 0,7 & 0.3 & - & 0,4 & 0,1 & 0,1 & - & 0,1 \\
\hline 26 & 0,7 & - & 0,3 & - & 0,2 & - & 0,2 & - \\
\hline 28 & 0,9 & 0,4 & - & - & 0,5 & - & - & - \\
\hline 30 & 1,1 & 0,3 & 0,6 & 0,3 & 0,5 & - & 0,4 & 0,1 \\
\hline
\end{tabular}

Blattfeder aus Stall $a=0,01$ ein; $b=1,5 \mathrm{~cm}$

\begin{tabular}{|c|c|c|c|c|c|c|c|c|c|c|}
\hline $\begin{array}{c}\text { Klemmbacken } \\
\text { aus Stahl }\end{array}$ & $\mathbf{1}$ & $\mathbf{1 , 8 8}$ & $\mathbf{2 , 2 2}$ & $\mathbf{3 , 0 0}$ & $\mathbf{3 , 6 2}$ & 4,30 & $\mathbf{6 , 2 5}$ & & & \\
\hline $\begin{array}{c}\text { Klemmbacken } \\
\text { aus Messing }\end{array}$ & $\mathrm{0,13}$ & $\mathbf{0 , 3 6}$ & $\mathbf{0 , 4 1}$ & $\mathbf{0 , 3 1}$ & $\mathbf{0 , 4 8}$ & $\mathbf{0 , 6 0}$ & & & \\
\hline & $\mathbf{1 , 4 5}$ & $\mathbf{1 , 9 2}$ & $\mathbf{2 , 3 5}$ & $\mathbf{2 , 9 0}$ & $\mathbf{3 , 4 8}$ & $\mathbf{4 , 2 7}$ & 5,12 & 5,83 & $\mathbf{6 , 1 0}$ \\
\hline
\end{tabular}

ger Institutes hinsichtlich ihrer Vergrösserungs- und Phasenverschiebungskurve geprïft. Bei Z10 waren die Blattfedem zwischen Stahlbezw. Aluminiumbacken geklemmt, bei $Z 11$ zwischen Aluminiumbezw. Messingbacken und bei $Z 12$ zwischen Stahl- bezw. ausgeglühte Messingbacken. Ausserdem wurde bei $Z 12$ noch die Länge der Blattfedern variiert. In Fig. 6-9 sind einige der aufgenommen Resonanzund Phasenkurven wiedergegeben. Bei den Resonanzkurven ist als Ordinate die Gesamtvergrösserung $V_{s} V_{d}$ aufgetragen.

Die Resonanz- und Phasenkurven zeigen in grossen und ganzen den für Blattfederseismographen charakteristischen Verlauf. In den Einzelheiten treten noch allerlei Abweichungen auf. Darauf soll jedoch hier nicht näher eingegangen werden, da sie meist nebensächli- 
cher Natur sind. Es sollen nur die wesentlichen Punkte hervorgehoben werden.

Für die statische Vergrösserung ergibt sich bei diesen Seismographen aus den Versuchen rund der Wert 10.000. (Der kleinste Wert, den die Resonanzkurve zwischen den beiden Koppelfrequenzen bei nicht zu starker Dämpfung erreicbt, ist die statische Vergrösserung).

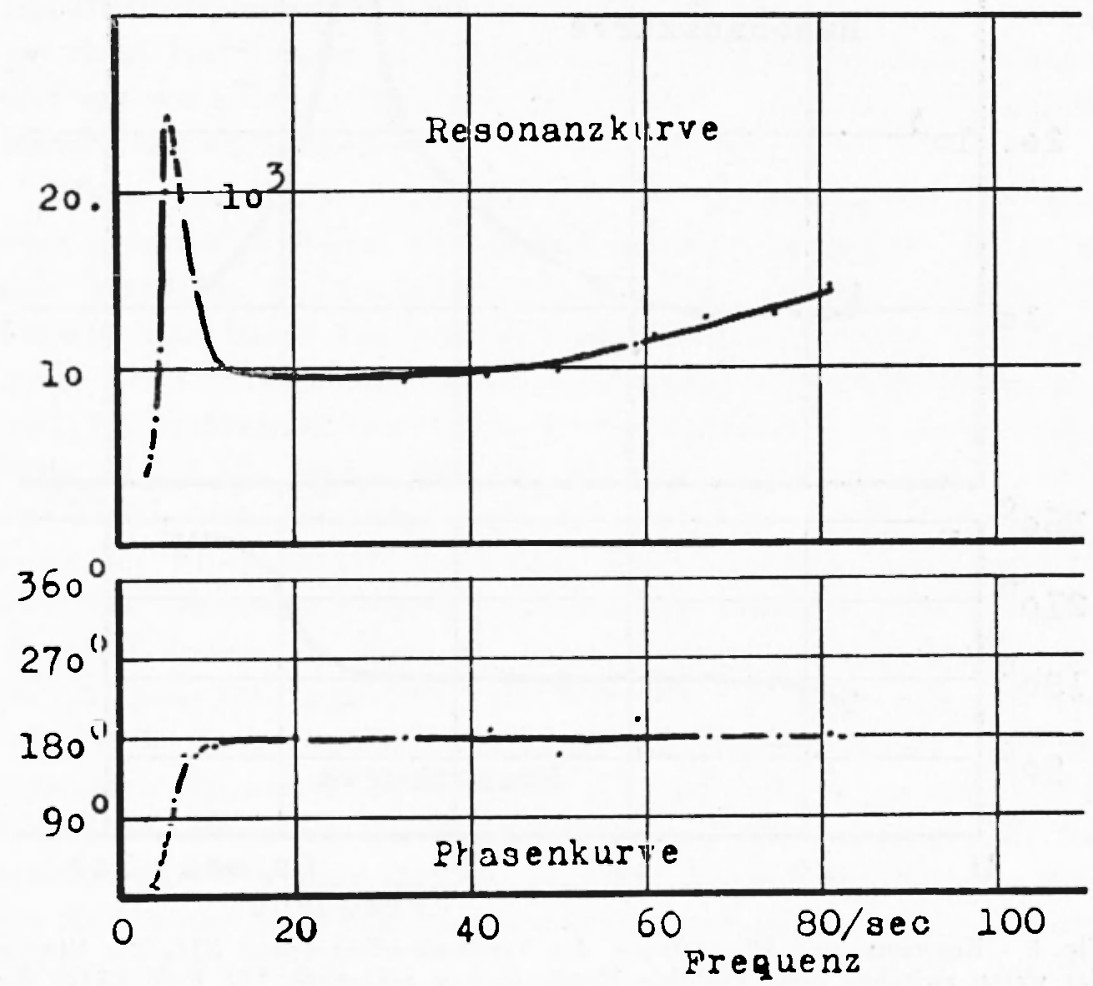

Fig. 7 - Resonanz- und Phasenkurve des Vertikalseismographen Z12. Die Blattfedern waren zwischen Stahlbacken geklemmt. Die freie Länge der Blattfedern betrug $0,02 \mathrm{~cm}$.

Da die optiscbe Vergrösserung durch die Drehspiegelachse bei $1 \mathrm{~m}$ Lichtweg 4000 fach ist, (auf diese Registrierentfernung sind alle Resonanzkurven bezogen), bleibt für die mechanische Verọ̆össerung durch den ersten Hebelarm der Wert 2,5. Die Länge des Hebelarnes beträgt $J_{1}=11,5 \mathrm{~cm}$. Für den Abstand der Achsen der beiden Koppelschwingungen ergibt sich daraus $L=J / V=11,5 / 2,5=4,6 \mathrm{~cm}$. Theoretisch berechnet man aus den Daten der zylinderfömigen Seismographenmasse olıne Beruicksichtigung der Dämpfungs- und Regi- 
striereinrichtung für $L$ die Zahl $2,7 \mathrm{~cm}$. Die Achse der zweiten Koppelschwingung, um die sich der Seismograph bei der erzwungenen Schwingung dreht, rïckt also wahrscheinlich durch die erwähnten Einrichtungen um ein beachtliches Stück über den theoretisch zu erwartenden Abstand hinaus von den Blattfedern weg.

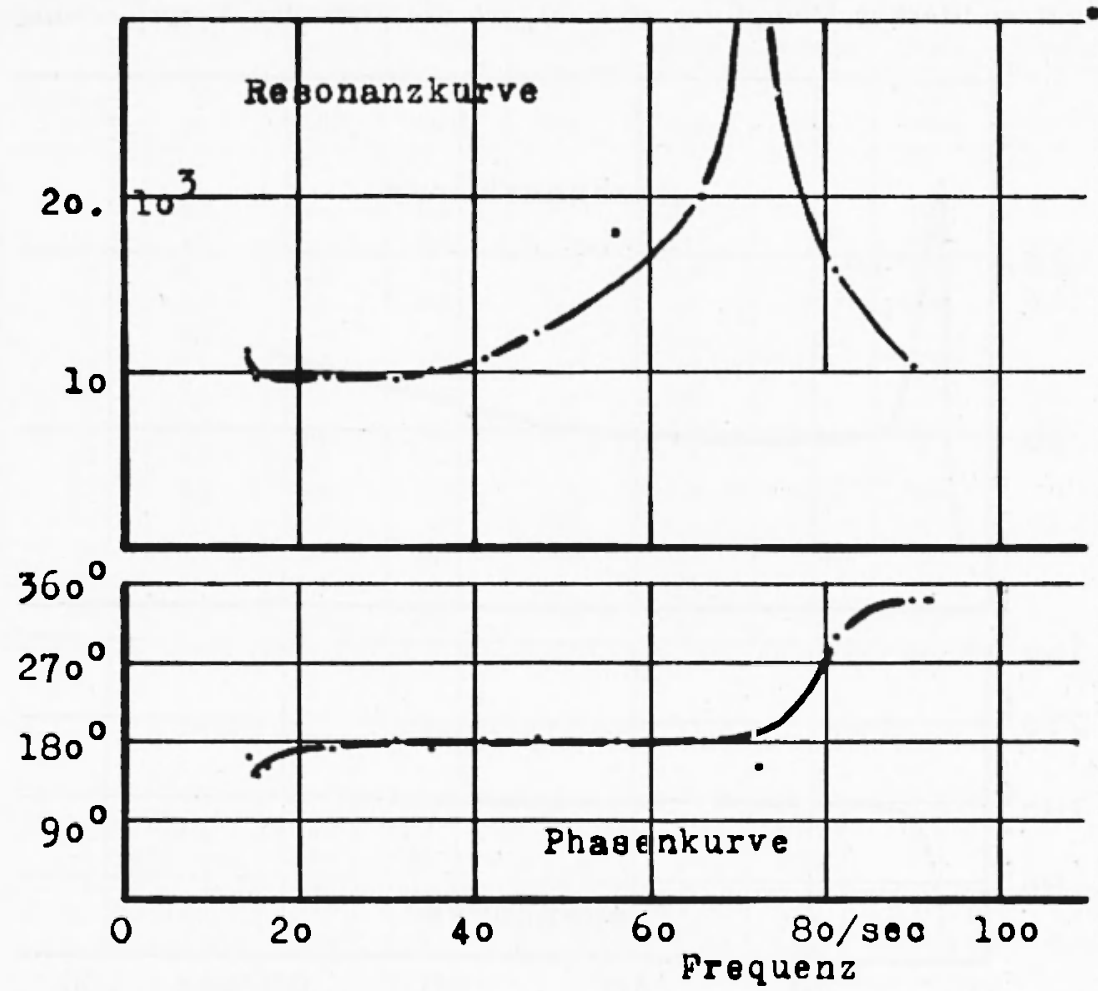

Fig. 8 - Resonanz- und Phasenkurve des Vertikalseismographen Z11. Die Blattfeder waren zwischen nicht geglühte Messingbacken geklemmt. Die freie Länge der Blattfedern betrug $0,02 \mathrm{~cm}$.

Von besonderem Interesse bei Blattfederseismographen ist die zweite Koppelfrequenz. Nach der theoretischen Berechnung auf Grund der Formeln, die im ersten Teil der Arbeit abgeleitet worden sind, müsste sie fir die drei untersuchten, gleichgebauten Apparate ungefähr $300 \mathrm{~Hz}$ sein. In Wirklichkeit ist sie aber wesentlich geringer. Meist ist sie sogar geringer als $100 \mathrm{~Hz}$. Die Ursache hierfür ist nach den Versuchsergebnissen in der Einspannung der Blattfedern zu suchen. Allerdings ist es hier so, dass man mit Klemmbacken aus Stahl und Aluminium eine bessere Annäherung an die theoretischen Werte 
bekommt als mit solchen aus ungeglühtem Messing. Die Verhältnisse liegen, soweit sich dies aus den Messungen schliessen lässt, gerade umgekehrt wie bei den freien Eigenschwingungen von einseitig eingespannten Stäben und Blattfedern. Wahrend bei diesen Blei- und Messingklemmbacken geeigneter sind als solcbe aus Aluminium und Stahl, ist bei erzwungenen Schwingungen die zweite Koppelfrequenz von Blattfederseismographen am grössten bei einer Klemmvorrichtung aus Stahl. Ungünstiger ist Aluminium und Messing in Normalzustand und am wenigsten geeignet ist nach obigen Versuchen ausgeglühtes Messing als Klemmbacken.

Dieser qualitativen Abschätzung des Einspannwertes soll kurz eine quantitative folgen. Die freie Länge der Blattfeder beträgt bei den benutzten Seismographen, falls nichts anderes erwähnt ist, $l=0,02 \mathrm{~cm}$. Damit bei idealer Einspannung die zweite Koppelfrequenz bei $n=70 \mathrm{~Hz}$ liegt, müsste die Blattfeder ungefähr $0,2 \mathrm{~cm}$ lang sein. Im vorhergehenden Abschnitt wurde für die wirksame Federlänge $(i+\bar{z} \dot{\Delta} i)$ gesetzt. Bei den Seismographen haben wir ausser der Klemmstelle an Stativ noch eine zweite an der Masse. Dadurch kann der Einspannwert vergrössert, möglicherweise verdoppelt werden. Die wirksame Federlänge kann also zwischen $(l+2 \Delta l)$ und $(l+4 \Delta l)$ liegen. Da diese zu $0,2 \mathrm{~cm}$ berechnet wurde, ergibt sich für $\Delta l$ ganz roh abgeschätzt der Wert $0,05 \mathrm{~cm}$. Führt man die entsprechende Rechnung für die erste Koppelfrequenz, die bei den untersuchten Apparaten um $v_{1}=4 H z$ schwankt, durch, so erhält man für $\Delta l$ nur etwa $l / 10$ des obigen Wertes. Es scheint, als ob $\Delta l$ mit zunehmender Frequenz ebenfalls zunimmt. Da mit der Frequenz auch die Krafte und Energien des Schwingungssystems zunehmen, ist dieser Befund erklärlich.

Aus der Resonanzkurve und der Lage der beiden Koppelfrequenzen ergibt sich, dass der Frequenzbereich, in welchem die geprüften Vertikalseismographen braucblbar arbeiten, zwischen $1 \mathrm{H}$ und $80 \mathrm{~Hz}$ liegt, sofern es allerdings gelingt, die zweite Koppelfrequenz durch

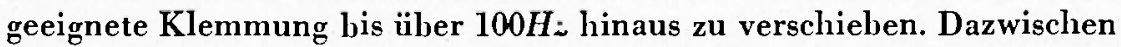
gibt es bei richtiger Einstellung der Dämpfung einen weiten Bereich, in welchem die Apparate mit praktiscb konstanter Vergrösserung arbeiten. Dadurch sind sie in der Lage, die meisten künstlich erregten Bodenerschïtterungen, wie solcbe, die durch grosse und kleine Sprengungen, durch Maschinen und Verkehr erzeugt werden, zienlich wabrheitsgetreu aufzuschreiben. 
3. Zusammenfassung der experimentellen Untersuchungen.

Die Prüfungen der untersuchten Blattfederseismographen haben ergeben, dass sich diese so verhalten, wie es die im ersten Teil entwickelte Theorie verlangt. Die Vergrösserungskurve besitzt zwei Maxima, zwei Resonanzstellen, die den beiden Koppelfrequenzen entspre-

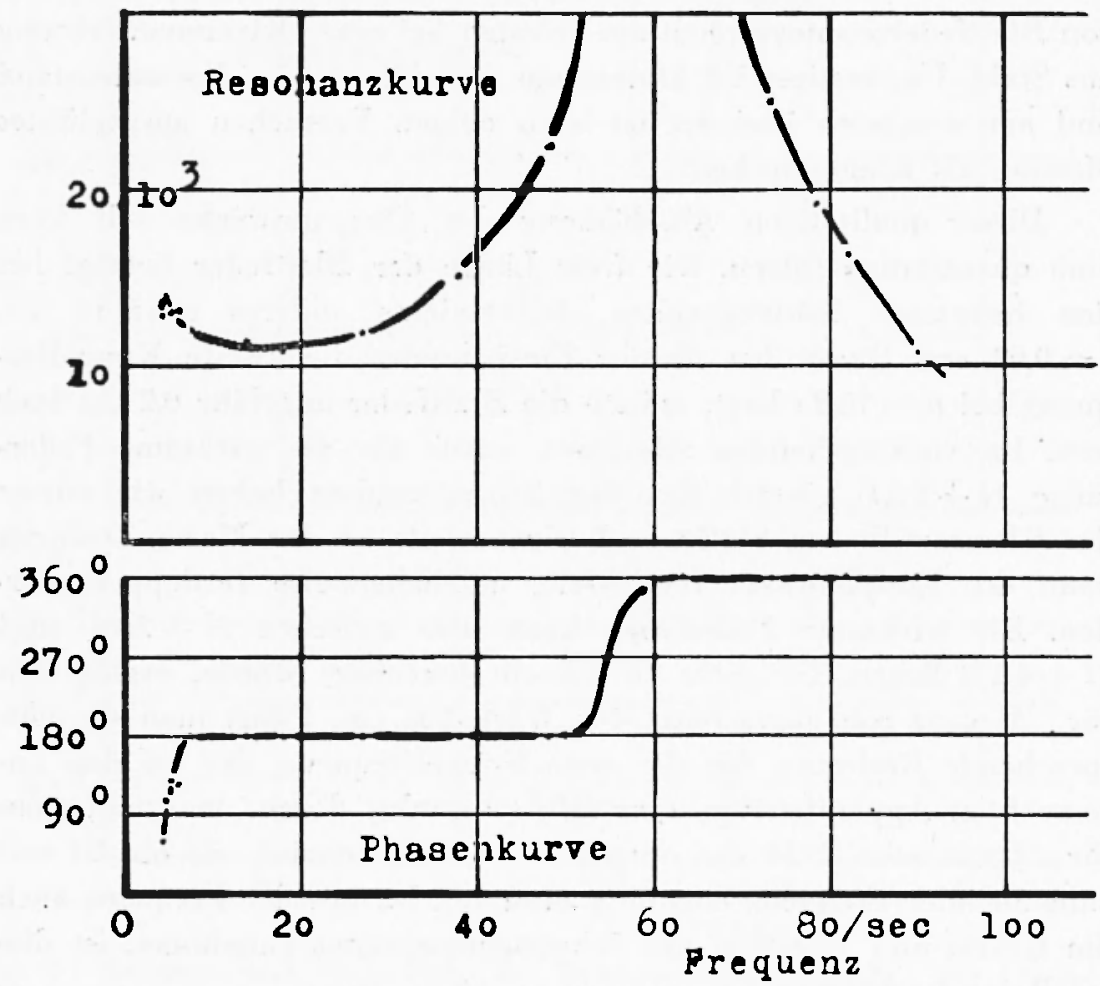

Fig. 9 - Resonanz- und Phasenkurve des Vertikalseismographen Z10. Die Blattfedern waren zwischen Aluminiumbacken geklemmt. Die freie Lïnge der Blattfeder betrug $0,02 \mathrm{~cm}$.

chen. Bei diesen Frequenzen springt die Phasenkurve jeweils um $180^{\circ}$. Bei den Frequenzen der Koppelschwingungen treten häufig erhelbliche Differenzen zwischen den theoretisch zu erwartenden und den experimentell gemessenen Werten auf. Diese Differenzen werden teils durch irgendwelche Vorbelastungen der Blattfedern verursacht, die zu einer Herabsetzung der Biegesteifigkeit führen, teils rühren sie von der nie völlig idealen Einklemmung der Blattfedern ber. Diese macht sich besonders bei der zweiten Koppelfrequenz unangenehm hemerkbar, da die unvollkommene Einspannung eine relativ grosse 
wirksame Länge der Blattfedern bewirkt, wodurch wiederum die Frequenz der zweiten Koppelsehwingung soweit herabgedrückt wird, dass sie in den Arbeitsbereich der Seismographen fällt. Die Versuche haben ergeben, dass man fur die Klemmbacken der Blattfedern ein Material mit möglichst hoher Schallgeschwindigkeit, wie Stahl, verwenden soll, um die zweite Koppelfrequenz nach relativ hohen Werten zu verschieben. Um bei statischer Belastung oder bei freien Eigenschwingungen von eingespannten Stäben und Balken der ideal festen Einspannung an nächsten zu kommen, muss man umgekehrt als Klemmbacken ein Material möglichst niedriger Schallgeschwindigkeit, wio etwa Blei, wählen.

\section{ZUSAMMENF ASSUNG}

In Weiterführung einer Arbeit von Rössiger wird sowohl durch theoretische Berechnung wie durch Untersuchungen nachgewiesen, dass Blattfederseismographen keine einfachen Schwingungsgebilde sind, sondern zwei Freiheitsgrade, und damit auch zwei Eigenfrequenzen besitzen, die sehr stark miteinander gekoppelt sind. Dadurch entstehen aus den Eigenfrequenzen zuei Koppelfrequenzen. Die Resonanzkurve weist zuei Resonanzstellen auf, von denen nur die erste durch die üblichen Dämpfungsvorrichtungen weggedämpft werden kann.

Die Untersuchungen haben ergeben, dass das Verhalten von Blattfederseismographen sehr stark durch irgendwelche Vorbelastungen and durch die Art der Einklemmung beeinflusst wird. Durch die Vorbelastung kann die Direktionshraft erhöht und erniedrigt werden. Der idealen Einklemmung von Blattfedern und Stäben kommt man am nïchsten, wenn man bei der Untersuchung von Eigenschwingungen und statischer Belastung Klemmaterial mit möglichst geringer Schallgeschwindigheit benutzt. Umgehehrt uird die zweite Koppelfrequenz bei Vervendung von Klemmaterial moglichst hoher Schallgeschwindigkeit ueit nach höheren Frequenzen hin verschoben, was anzustreben ist.

\section{SUMMARY}

In continuing a uork of $R \ddot{o s s i g e r}$ it is demonstrated as uell by theory as by experiment that plate-spring seismographs are no sim- 
ple vibration-system, but have two degrees of freedom and therefore two free vibrations that are very strongly coupled together. Thereby two coupled frequencies result of the free vibration-periods. The resonance-curve shows two resonance-points at these frequencies of which only the first one can be damped by the damping apparatus. The experiments have demonstrated that the behaviour of the plate-spring seismograph is strongly influenced by any other charge und by the kind of fastening. By other charge the directive force can be increased or decreased. We approach us best to the ideal fastening of plate-springs and bars, if we use at the analysis of free vibrations and static deflection fastening material with a velocity of sound as low as possible. Opposite, if there is used a fastening material of a velocity of sound as high as possible, the second coupledfrequency is widely displaced to higher frequencies what should be uanted.

\section{LITERATURVERZEICHNIS}

(1) Rössigrer M., Die elastischen Schuingungen einer Masse, die durch eine Blatteder gehalten wird. Annalen d. Physik, 5. Folge, 15. Bd. (1932), Nr. 6 S. 735.

(2) Rossigen M, Beitrag zur Theorie des Blattfederseismographen. Zeitschr. f. Geophysik, Bd. VIII (1932), S. 470.

(3) Angenheister G., Bodenschwingingen. Ergebnisse der exakten Naturwissenschaften, Bd. XV (1936), S. 310.

(4) Schulze G. A. und Fortsch O., Die seismischen Beobachtungen bei der Sprengung auf Helgoland am 18. April 1947 zur Erforschung des tieferen Untergrundes. Geologisches Jahrbuch 64, Hannover-Celle 1950. S. 204.

(5) KoHLER R. Eine neue Methode der Seismographenprïfung. Zeitschr. f. Geophysik, Bd. VIII (1932), S. 74.

(6) Föвтsch O., Das Verhnlten noch freistehender Brückenpleiler bei Schwingungen und deren Abhängigkeit vom Untergrund. Zeitschr. f. Geophysik, Bd. XIV (1938), S. 173.

(7) “ Hiitte », des Ingenieurs Taschenbuch I, 27. Auf. (1949), S. 236. 